\title{
A splitting result for the algebraic $K$-theory of projective toric schemes
}

\author{
Thomas Hüttemann
}

Received: 9 March 2011 / Accepted: 26 October 2011 / Published online: 13 March 2012

(C) Tbilisi Centre for Mathematical Sciences 2012

\begin{abstract}
Suppose $X$ is a projective toric scheme defined over a ring $R$ and equipped with an ample line bundle $\mathcal{L}$. We prove that its $K$-theory has a direct summand of the form $K(R)^{(k+1)}$ where $k \geqslant 0$ is minimal such that $\mathcal{L}^{\otimes(-k-1)}$ is not acyclic. Using a combinatorial description of quasi-coherent sheaves we interpret and prove this result for a ring $R$ which is either commutative, or else left NOETHERian.
\end{abstract}

Keywords Toric scheme - Quasi-coherent sheaf - Perfect complex · Algebraic $K$-theory

Mathematics Subject Classification Primary 18F25; Secondary 14F05 . $18 \mathrm{~F} 20 \cdot 18 \mathrm{G} 35$

\section{Contents}

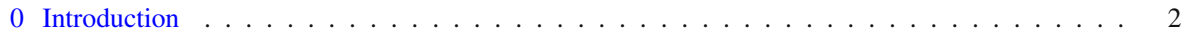

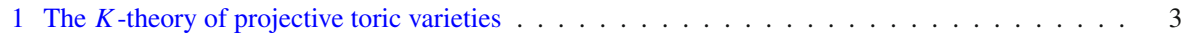

1.1 Complexes of sheaves on projective toric varieties . . . . . . . . . . . . . . 3

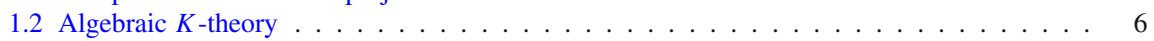

1.3 Justification of terminology . . . . . . . . . . . . . . . . . . . . . . . 7

1.4 Lattice points and Ehrhart polynomials . . . . . . . . . . . . . . . . . . . . 8

Communicated by Michael Weiss.

This work was supported by the Engineering and Physical Sciences Research Council [grant number EP/H018743/1].

T. Hüttemann $(\varangle)$

Pure Mathematics Research Centre, Queen's University Belfast,

Belfast BT7 1NN, Northern Ireland, UK

e-mail: t.huettemann@qub.ac.uk 


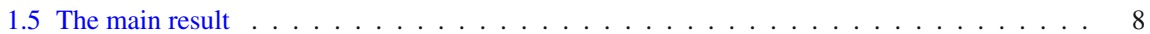

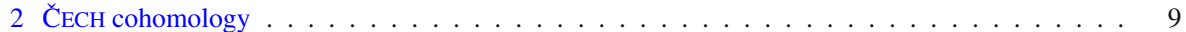

2.1 Double complexes and the spectral sequence argument . . . . . . . . . . . . . . . 9

2.2 ČECH cohomology . . . . . . . . . . . . . . . . . . . . . . . . . . . 10

2.3 Line bundles determined by $P \ldots \ldots \ldots \ldots \ldots \ldots \ldots \ldots$

2.4 Twisting sheaves . . . . . . . . . . . . . . . . . . . . . . . . 12

2.5 Quasi-coherent functors . . . . . . . . . . . . . . . . . . . . . . . . . . . . . . . . . .

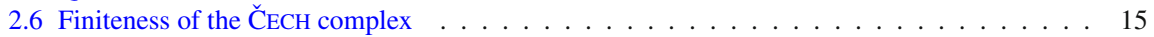

2.7 Canonical sheaves and suspension of chain complexes . . . . . . . . . . . . . . . 15

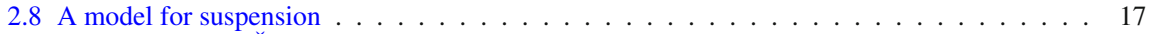

2.9 Relating $\mathcal{O}(0) \otimes \check{\Gamma}$ to $n$th suspension . . . . . . . . . . . . . . . . . . . . . . . . . 20

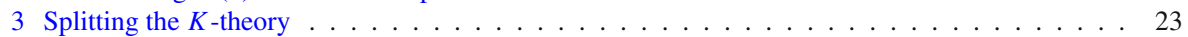

3.1 Reduced $K$-theory . . . . . . . . . . . . . . . . . . . . . . . . . . 23

3.2 Further splitting . . . . . . . . . . . . . . . . . . . . . . . . 24

3.3 Algebraic $K$-theory of projective space . . . . . . . . . . . . . . . . . . 27

\section{Introduction}

Let $R$ be a commutative ring with unit. An $n$-dimensional polytope $P \subseteq \mathbb{R}^{n}$ with integral vertices determines a projective toric $R$-scheme $X_{P}$ together with a family of line bundles $\mathcal{O}_{X_{P}}(k)$ (a family of quasi-coherent sheaves of $\mathcal{O}_{X_{P}}$-modules which are locally free of rank 1). To a given chain complex of quasi-coherent sheaves $\mathcal{F}_{\bullet}$ we associate an $R$-module chain complex $\check{\Gamma}\left(\mathcal{F}_{\bullet}\right)$ given by first forming a ČECH complex in each chain degree of $\mathcal{F}_{\bullet}$, and then taking the total complex of the resulting twofold chain complex.

Set $n_{P}=0$ if $P$ has integral points in its interior; otherwise, let $n_{P} \geqslant 1$ be such that the dilated polytope $\left(n_{P}+1\right) P$ has lattice points in its interior, but $n_{P} P$ does not. This number can be characterised in different ways: $n_{P} \geqslant 0$ is minimal among non-negative integers $k$ such that $\mathcal{O}_{X_{P}}(-k-1)$ is not acyclic; also, $n_{P}$ is the number of distinct integral roots of the EHRHART polynomial of $P$.

We will show that there is a homotopy equivalence of $K$-theory spaces

$$
K\left(X_{P}\right) \simeq \underbrace{K(R) \times \cdots \times K(R)}_{n_{P}+\text { ffactors }} \times K\left(X_{P},\left[n_{P}\right]\right)
$$

where $K$-theory is defined using perfect complexes of sheaves and modules, and the last factor on the right denotes the $K$-theory of those perfect complexes $\mathcal{F}_{\bullet}$ of quasicoherent sheaves on $X_{P}$ for which $\check{\Gamma}\left(\mathcal{F}_{\bullet}(k)\right)$ is acyclic for $0 \leqslant k \leqslant n_{P}$. In fact, we will prove slightly more: by exploiting a strictly combinatorial viewpoint of sheaves on toric varieties we can prove the corresponding result for a unital ring $R$ which is commutative, or else left NOETHERian.

A corresponding result has been proved by the author in a "non-linear" context, replacing modules by topological spaces [5]. It must also be pointed out that the splitting result is, in general, far from optimal: a lot of $K$-theoretical information can be left over in the factor $K\left(X_{P},\left[n_{P}\right]\right)$. For example, if $n_{P}=0$ (which can be guaranteed by first replacing $P$ by its dilate $(n+1) P$ ) the splitting results merely gives a version of reduced $K$-theory. But in the other extreme, if $P$ is an $n$-dimensional simplex with volume $1 / n$ ! then $X_{P}=\mathbb{P}^{n}$ is $n$-dimensional projective space; one can show 
that $n_{P}=n$ and $K\left(\mathbb{P}^{n},[n]\right) \simeq *$ in this case so that we recover the known splitting results for projective spaces, generalised to suitable non-commutative ground rings (Theorem 3.3.1). There are, of course, intermediate cases; suffice it to mention that 0/1-polytopes do not have interior lattice points and hence lead to non-trivial splittings with $n_{P} \geqslant 1$. Interesting examples of $0 / 1$-polytopes arise as STANLEY's order and chain polytope associated to finite posets [10].

The paper is divided into three parts. In Sect. 1 we introduce the combinatorial framework for sheaves on projective toric varieties, and give a first formulation of the main result, Theorem 1.5.1. We also prove that for a commutative ring $R$ we recover the usual notions of algebraic geometry. In Sect. 2 we develop some algebraic geometry from the combinatorial viewpoint, allowing for a non-commutative ground ring $R$ : we define twisting sheaves and study the ČECH complex of various of complexes of sheaves. Of major importance is the finiteness Theorem 2.6.1 which asserts that the ČECH complex of a perfect complex is a perfect complex of $R$-modules. In the left NOETHERian case this is quite straightforward, while the non-NOETHERian commutative case requires NOETHERian approximation (descent). Finally, Sect. 3 contains a detailed formulation of the main theorem, and its proof.

We assume some familiarity with basic homological algebra as presented by WEIBEL [13], WALDHAUSEN $K$-theory [12] and its formulation in an algebro-geometric setting by THOMASON and TROBAUGH [11]. We mention a few conventions used in this paper. Chain complexes are topologically indexed: differentials lower the degree by 1 . If needed, modules are considered as chain complexes concentrated in chain degree 0 . The term "module" without any qualification refers to a left module.

\section{The $K$-theory of projective toric varieties}

\subsection{Complexes of sheaves on projective toric varieties}

We start by a combinatorial description of quasi-coherent sheaves on toric schemes defined by a polytope.

Let $P \subseteq \mathbb{R}^{n}$ be an $n$-dimensional polytope with integral vertices. Each non-empty face $F$ of $P$ gives rise to a cone (the set of finite linear combinations with non-negative real coefficients)

$$
T_{F}^{P}=T_{F}=\text { cone }\{p-f \mid p \in P, f \in F\},
$$

the tangent cone of $F$, and hence to an additive monoid $S_{F}^{P}=S_{F}=T_{F} \cap \mathbb{Z}^{n}$.

Lemma 1.1.1 Let $F \subseteq G$ be a pair of non-empty faces of $P$. Then there exists a vector $v_{F, G} \in \mathbb{Z}^{n} \cap T_{F}$ such that

$$
T_{G}=T_{F}+\mathbb{R} v_{F, G} \quad \text { and } \quad S_{G}=S_{F}+\mathbb{Z} v_{F, G}
$$

In the former case the symbol "+" denotes MINKOWSKI sum, the set of sums of elements of the indicated cones; in the latter, the symbol "+" denotes the submonoid of $\mathbb{Z}^{n}$ generated by sums of elements of the indicated monoids. 
Proof This is Proposition 1.2.2 of [2], applied to the dual cones $\sigma=T_{F}^{\vee}$ and $\tau=T_{G}^{\vee}$.

Now let $R$ denote an arbitrary ring with unit. We then have monoid $R$-algebras $A_{F}^{P}=A_{F}=R\left[S_{F}\right]$ for every non-empty face $F$ of $P$; an element $v \in S_{F}$ gives rise to the element $x=1 \cdot v \in A_{F}$. Note that $A_{F}$ is not commutative unless $R$ is, but that the element $x$ lies in the centre of $A_{F}$. Moreover, monoid generators of $S_{F}$ give rise to $R$-algebra generators of $A_{F}$ which is thus a finitely generated algebra by GoRDAN's Lemma ([2,1.2.1], applied to the dual cone $\left.\sigma=T_{F}^{\vee}\right)$. The previous Lemma implies immediately:

Lemma 1.1.2 For $F \subseteq G$ the algebra $A_{G}$ is obtained from the algebra $A_{F}$ by localising by a single element $x_{F, G}$ in the centre of $A_{F}$. Consequently, for every $p \in A_{G}$ there is $N \geqslant 0$ such that $x_{F, G}^{N} \cdot p \in A_{F}$.

Replacing $P$ by an integral dilate $k P=\{k p \mid p \in P\}, k \geqslant 1$, and replacing the face $F$ by its dilate $k F$ does not change these cones, monoids and algebras. That is, $T_{F}^{P}=T_{k F}^{k P}$, and similarly for $S_{F}$ and $A_{F}$.

Definition 1.1.3 (Presheaves) Let $F(P)_{0}$ denote the set of non-empty faces of $P$, partially ordered by inclusion. We write $\mathrm{Ch}(R)$ for the category of (possibly unbounded) chain complexes of $R$-modules.

1. A presheaf on $P$ is a functor

$$
Y: F(P)_{0} \longrightarrow \mathrm{Ch}(R), F \mapsto Y^{F}
$$

equipped with extra data which turns the object $Y^{F}$ into a chain complex of (left) $A_{F}$-modules, such that for each pair of non-empty faces $F \subseteq G$ of $P$ the structure map $Y^{F} \longrightarrow Y^{G}$ is a map of $A_{F}$-module chain complexes.

2. A map of presheaves is a natural transformation of functors such that its $F$-component is an $A_{F}$-linear map, for each $F \in F(P)_{0}$. The category of presheaves is denoted by Pre $(P)$.

3. A map $f: Y \longrightarrow Z$ of presheaves is called a quasi-isomorphism, or a weak equivalence, if all its components $f^{F}$ are quasi-isomorphisms of chain complexes of modules.

\section{Definition 1.1.4 (Quasi-coherent sheaves)}

1. An object $Y \in$ Pre $(P)$ is called a quasi-coherent sheaf, or sheaf for short, if for each pair of non-empty faces $F \subseteq G$ of $P$, the adjoint structure map

$$
A_{G} \otimes_{A_{F}} Y^{F} \longrightarrow Y^{G}
$$

is an isomorphism of $A_{G}$-module chain complexes.

2. The full subcategory of Pre $(P)$ consisting of sheaves is denoted qCoh $(P)$. 


\section{Definition 1.1.5 (Homotopy sheaves)}

1. A presheaf $Y \in$ Pre $(P)$ is called a homotopy sheaf if for each pair of non-empty faces $F \subseteq G$ of $P$ the adjoint structure map $A_{G} \otimes_{A_{F}} Y^{F} \longrightarrow Y^{G}$ is a quasi-isomorphism of chain complexes.

2. The full subcategory of Pre $(P)$ consisting of homotopy sheaves is denoted hCoh $(P)$.

Every sheaf is a homotopy sheaf, and qCoh $(P)$ is a full subcategory of hCoh $(P)$. Moreover, the notion of a homotopy sheaf is homotopy invariant in the following sense: If $f: Y \longrightarrow Z$ is a weak equivalence of presheaves, then $Y$ is a homotopy sheaf if and only if $Z$ is a homotopy sheaf. This is true since $A_{G}$ is a localisation of $A_{F}$ by 1.1.2, for every pair of non-empty faces $F \subseteq G$ of $P$, so that the functor $A_{G} \otimes_{A_{F}}-$ is exact.

A chain complex of modules over some ring is called strict perfect if it is bounded and consists of finitely generated projective modules. It is called perfect if it is quasiisomorphic to a strict perfect complex. In fact, a complex $C$ is perfect if and only if there exists a strict perfect complex $B$ and a quasi-isomorphism $B \longrightarrow C$.

\section{Definition 1.1.6 (Perfect complexes and vector bundles)}

1. The homotopy sheaf $Y \in$ hCoh $(P)$ is called a perfect complex if for each $F \in$ $F(P)_{0}$ the chain complex $Y^{F}$ is a perfect complex of $A_{F}$-modules.

2. The full subcategory of hCoh $(P)$ consisting of perfect complexes is denoted by Perf $(P)$.

3. A homotopy sheaf $Y \in \mathrm{hCoh}(P)$ is called a homotopy vector bundle if for each $F \in F(P)_{0}$ the chain complex $Y^{F}$ is a strict perfect complex of $A_{F}$-modules.

4. The full subcategory of hCoh $(P)$ consisting of homotopy vector bundles is denoted hVect $(P)$.

The notion of a perfect complex is homotopy invariant in the manner described above for homotopy sheaves.

In algebraic geometry, a perfect complex can be replaced up to quasi-isomorphism by a bounded chain complex of vector bundles provided the scheme under consideration has an ample line bundle (as is the case for a projective scheme) or, more generally, has an ample family of line bundles. In the homotopy world the replacement is possible without reference to such additional structure.

Lemma 1.1.7 For $Y \in \operatorname{Perf}(P)$ there is a homotopy vector bundle $Z \in \operatorname{hVect}(P)$ together with a quasi-isomorphism $\zeta: Z \stackrel{\cong}{\rightarrow} Y$.

Proof By definition of perfect complexes there is, for each $F \in F(P)_{0}$, a bounded chain complex $V^{F}$ of finitely generated $A_{F}$-modules together with a quasi- isomorphism $v^{F}: V^{F} \longrightarrow Y^{F}$.

Let $\mathcal{P}(d)$ denote the sub-poset of $F(P)_{0}$ of faces of dimension at most $d$. For $F$ a vertex of $P$ define $Z^{F}=V^{F}$ and $\zeta^{F}=v^{F}$; this defines a $\mathcal{P}(0)$-diagram $Z$ and a quasi-isomorphism $\left.Z \longrightarrow Y\right|_{\mathcal{P}(0)}$. 
Suppose we have constructed a quasi-isomorphism of $\mathcal{P}(d-1)$-diagrams $Z \longrightarrow$ $Y_{\mathcal{P}(d-1)}$ such that each component of $Z$ is a strict perfect complex over the appropriate algebra. We show how to extend this data to $\mathcal{P}(d)$.

Fix a $d$-dimensional face $F$ of $P$, and let

$$
L^{F} Z=\underset{\substack{G \subseteq F \\ \operatorname{dim} G<d}}{\operatorname{colim}} A_{F} \otimes_{A_{G}} Z^{G} .
$$

This is a strict perfect complex of $A_{F}$-modules. We define $L^{F} Y$ by a similar colimit with $Z^{G}$ replaced by $Y^{G}$. These come equipped with canonical maps

$$
L^{F} Z \longrightarrow L^{F} Y \longrightarrow Y^{F}
$$

induced by the maps $\zeta^{G}$ defined before, and the structure maps of $Y$. Up to homotopy, the composition factors over the quasi-isomorphism $v^{F}$ (this follows from [13, 10.4.7] together with the fact that $v^{F}$ induces an isomorphism of hom-sets $\operatorname{hom}\left(L^{F} Z, V^{F}\right) \cong$ hom $\left(L^{F} Z, Y^{F}\right)$ in the derived category of $\left.A_{F}\right)$. Let $Z^{F}$ be the mapping cylinder of $L^{F} Z \longrightarrow V^{F}$; a homotopy then determines a map $\zeta^{F}: Z^{F} \longrightarrow Y^{F}$ such that the two compositions

$$
L^{F} Z \longrightarrow Z^{F} \longrightarrow Y^{F} \quad \text { and } \quad L^{F} Z \longrightarrow L^{F} Y \longrightarrow Y^{F}
$$

agree. Since $Z^{F} \simeq V^{F}$ the map $\zeta^{F}$ is a quasi-isomorphism. For $G \subset F$ define a structure map $Z^{G} \longrightarrow Z^{F}$ as the composition $Z^{G} \longrightarrow L^{F} \longrightarrow Z^{F}$, considered as maps of $A_{G}$-modules.

Performing this construction for each $d$-dimensional face of $P$ yields a $\mathcal{P}(d)$-diagram $Z$ together with a quasi-isomorphism $\zeta:\left.Z \longrightarrow Y\right|_{\mathcal{P}(d)}$. At the $n$th step we arrive at the assertion of the Lemma.

\subsection{Algebraic $K$-theory}

The category Perf $(P)$ carries the structure of a "complicial biWALDHAUSEN category" in the sense of THOMASON and TROBAUGH [11, 1.2.11]; as ambient ABELian category we choose the category Pre $(P)$. The weak equivalences are as defined in 1.1.3. The cofibrations are the degreewise split injections $Y \longrightarrow Z$ in Perf $(P)$ with cokernel in Perf $(P)$.

Definition 1.2.1 The algebraic $K$-theory of $P$ is defined to be the $K$-theory space of the complicial biWALDHAUSEN category Perf $(P)$. In symbols,

$$
K(P)=\Omega\left|w \mathcal{S}_{\bullet} \operatorname{Perf}(P)\right|
$$

where the symbol " $w$ " denotes the subcategory of weak equivalences as usual. 


\subsection{Justification of terminology}

Let now $R$ be a commutative ring with unit. The polytope $P$ determines a projective $R$-scheme $X_{P}$, obtained from the affine schemes $U_{F}=\operatorname{Spec} A_{F}$ by gluing $U_{F}$ and $U_{G}$ along their common open subscheme $U_{F \vee G}$. A chain complex $\mathcal{F}$ of quasi-coherent $\mathcal{O}_{X_{P}}$-modules gives rise, by evaluation on open sets $U_{F}$, to a sheaf

$$
Y_{\mathcal{F}}: F \mapsto \Gamma\left(U_{F}, \mathcal{F}\right)
$$

as defined in 1.1.4. The categories of chain complexes of quasi-coherent $\mathcal{O}_{X_{P}}$-modules and of perfect complexes of quasi-coherent $\mathcal{O}_{X_{P}}$-modules in the sense of [11, 2.2.10] are equivalent, via this construction, to the categories qCoh $(P)$ and $\operatorname{Perf}(P) \cap$ $\mathrm{qCoh}(P)$, respectively.

Lemma 1.3.1 Every homotopy sheaf $Y \in$ hCoh $(P)$ can be functorially replaced by a chain complex of quasi-coherent $\mathcal{O}_{X_{P}}$-modules $\mathcal{F}$ in such a way that $\Gamma\left(U_{F}, \mathcal{F}\right)$ and $Y^{F}$ are quasi-isomorphic. More precisely, there exists a homotopy sheaf $\bar{Y} \in$ $\mathrm{hCoh}(P)$, and there exist maps $Y_{\mathcal{F}} \longrightarrow \bar{Y} \longleftarrow Y$, cf. (1.3.0.1), which restrict to quasi-isomorphisms of chain complexes on $F$-components for every $F \in F(P)_{0}$. Moreover, this data can be chosen to depend on $Y$ in a functorial manner.

Proof This is the content of [6, 4.4.1]. In short, the homotopy sheaf $\bar{Y}$ is a fibrant replacement of $Y$ with respect to a suitable model structure on Pre $(P)$ (the replacement can be chosen functorially in $Y$ ), and $\mathcal{F}$ is the limit of the $F(P)_{0}$-diagram of quasi-coherent $\mathcal{O}_{X_{P}}$-modules $F \mapsto j_{*}^{F}\left(\tilde{Y}^{F}\right)$. Here $j^{F}$ is the inclusion $U_{F} \subset X_{P}, j_{*}^{F}$ is push-forward along $j$, and $\tilde{Y}^{F}$ is the the chain complex of quasi-coherent $\mathcal{O}_{U_{F}}$-modules associated to the complex of modules $\bar{Y}^{F}$.

For $Y \in \operatorname{Perf}(P)$ the chain complex $\mathcal{F}$ of Lemma 1.3.1 is a perfect complex in the sense of $[11,2.2 .10]$. Conversely, every perfect complex $\mathcal{F}$ of quasi-coherent $\mathcal{O}_{X_{P}}$-modules gives rise to an object $Y_{\mathcal{F}}: F \mapsto \Gamma\left(U_{F}, \mathcal{F}\right)$ of Perf $(P)$, by $[11,2.4 .3]$ applied to the affine schemes $U_{F}$.

Definition 1.3.2 The algebraic $K$-theory $K\left(X_{P}\right)$ of $X_{P}$ is the algebraic $K$-theory space of the complicial biWALDHAUSEN category of perfect complexes of quasi-coherent $\mathcal{O}_{X_{P}}$-modules, equipped with weak equivalences the quasi-isomorphisms, and cofibrations the degreewise split monomorphisms with cokernel a perfect complex.

This is the "right" definition by $[11,3.6]$ since the scheme $X_{P}$ is quasi-compact (a finite union of affine schemes) and semi-separated (with open affine subschemes $U_{F}$ as semi-separating cover).

Proposition 1.3.3 The algebraic $K$-theory space $K\left(X_{P}\right)$ of $X_{P}$ is homotopy equivalent to $K(P)$.

Proof The functor sending a perfect complex $\mathcal{F}$ to its associated object $Y_{\mathcal{F}}$ of Perf $(P)$,

$$
Y_{\mathcal{F}}: F \mapsto \Gamma\left(U_{F}, \mathcal{F}\right)
$$


is exact and induces, in view of Lemma 1.3.1, an equivalence of derived categories. By $[11,1.9 .8]$ this implies that $K\left(X_{P}\right) \simeq K(P)$ as claimed.

\subsection{Lattice points and Ehrhart polynomials}

We need to introduce a classical result on counting lattice points in polytopes. Let $P \subseteq \mathbb{R}^{n}$ be an $n$-dimensional polytope with integral vertices. For a non-negative integer $k$, let $N_{P}(k)$ denote the number of integral points in the dilation $k P$ of $P$. Since $0 P=\{0\} \subset \mathbb{R}^{n}$ we have $N_{P}(0)=1$.

Theorem 1.4.1 (Ehrhart Theorem) There is a unique polynomial $E_{P}(x)$ with rational coefficients, called the EHRHART polynomial of $P$, such that $E_{P}(k)=N_{P}(k)$ for all non-negative integers $k$. The polynomial $E_{P}(x)$ has degree $n$, constant term 1 and leading coefficient vol $(P)$ (with volume normalised so that vol $\left.\left([0,1]^{n}\right)=1\right)$. Moreover, if $j$ is a negative integer then $(-1)^{n} E_{P}(j)$ is the number of integral points in the interior of the dilation $j P$.

The reader can find a proof of this remarkable theorem in $[7,12.16]$ or $[1, \S 18]$. From the geometric meaning of the EHRHART polynomial we deduce:

Corollary 1.4.2 All integral zeros of $E_{P}(x)$ are negative, and the set of integral zeros of $E_{P}(x)$ is of the form $\left\{-n_{P},-n_{P}+1, \ldots,-1\right\}$ for some integer $n_{P} \in[0, n]$. The number $n_{P}$ is minimal among integers $k \geqslant 0$ such that $-(k+1) P$ has integral points in its interior.

\subsection{The main result}

We can now formulate a preliminary version of the main result of this paper.

Theorem 1.5.1 Let $P \subseteq \mathbb{R}^{n}$ be an n-dimensional polytope with integral vertices, and let $n_{P}$ denote the number of distinct integral roots of its EHRHART polynomial. Let $R$ be a ring with unit. Suppose that $R$ is commutative, or else left NOETHERian. Then there is a homotopy equivalence of $K$-theory spaces

$$
K(P) \simeq K(R)^{n_{P}+1} \times K\left(P,\left[n_{P}\right]\right)
$$

where $K(R)$ denotes the $K$-theory space of the ring $R$, defined using perfect complexes of $R$-modules, and $K\left(P,\left[n_{P}\right]\right)$ denotes the $K$-theory space of a certain subcategory of the category Perf $(P)$.

In view of Proposition 1.3.3, this implies a splitting result for the algebraic $K$-theory of projective toric $R$-schemes provided $R$ is a commutative ring with unit.

The proof of Theorem 1.5.1 will be given in Sect. 3.2, and will contain explicit descriptions of the homotopy equivalence and of $K\left(P,\left[n_{P}\right]\right)$. 


\section{2 ČECH cohomology}

2.1 Double complexes and the spectral sequence argument

We work over an arbitrary unital ring $R$. Let $D_{*, *}$ be a double complex of $R$-modules; that is, we are given $R$-modules $D_{p, q}$ for $p, q \in \mathbb{Z}$, "horizontal" and "vertical" differentials

$$
\partial^{h}: D_{p, q} \longrightarrow D_{p-1, q} \quad \text { and } \quad \partial^{v}: D_{p, q} \longrightarrow D_{p, q-1}
$$

with $\partial^{h} \circ \partial^{h}=0$ and $\partial^{v} \circ \partial^{v}=0$, such that

$$
\partial^{h} \circ \partial^{v}=-\partial^{v} \circ \partial^{h} .
$$

Its total complex Tot $D_{*, *}$ is a chain complex with $\operatorname{Tot}\left(D_{*, *}\right)_{n}=\bigoplus_{p+q=n} D_{p, q}$ and differential $\partial=\partial^{h}+\partial^{v}$, cf. [13, 1.2.6].

Let $D_{*, *}^{\prime}$ be another double complex of $R$-modules. A map of double complexes $f: D_{*, *} \longrightarrow D_{*, *}^{\prime}$ is a collection of $R$-linear maps $D_{p, q} \longrightarrow D_{p, q}^{\prime}$ which commute with vertical and horizontal differentials.

Proposition 2.1.1 (The spectral sequence argument) Let $D_{*, *}$ be a double complex of $R$-modules. Suppose that $D_{*, *}$ is concentrated in the first $n+1$ columns so that $D_{p, *}=0$ if $p<0$ or $p>n$, or that $D_{*, *}$ is concentrated in the first $n+1$ rows so that $D_{*, q}=0$ if $q<0$ or $q>n$.

1. There is a convergent spectral sequence

$$
E_{p, q}^{1}=H_{q}^{h}\left(D_{*, p}\right) \Longrightarrow H_{p+q} \operatorname{Tot} D_{*, *}
$$

with $E_{p, q}^{2}=H_{p}^{v} H_{q}^{h}\left(D_{*, *}\right)$. Here $H^{h}$ denotes taking homology modules with respect to the horizontal differential $\partial^{h}$, and $H^{v}$ denotes taking homology modules with respect to the vertical differential $\partial^{v}$.

2. Suppose that $D_{*, *}^{\prime}$ is another double complex of $R$-modules concentrated in the first $n+1$ columns or rows. Suppose that the map of double complexes $f$ : $D_{*, *} \longrightarrow D_{*, *}^{\prime}$ induces an isomorphism on horizontal homology modules. Then $f$ induces a quasi-isomorphism

$$
\operatorname{Tot}(f): \text { Tot } D_{*, *} \stackrel{\simeq}{\rightarrow} \operatorname{Tot} D_{*, *}^{\prime} \cdot
$$

Proof The spectral sequence in (1) arises in the standard way from a filtration of Tot $D_{*, *}$ by the rows of $D_{*, *}$, see [13, Sect. 5.6] for details of the construction, and $[13,5.2 .5]$ for convergence. The result of (2) now follows from convergence of the bounded spectral sequences for $D_{*, *}$ and $D_{*, *}^{\prime}$, together with the fact that by hypothesis $f$ induces an isomorphism of spectral sequences on the $E^{1}$-term. 


\section{2 ČECH cohomology}

Assume now that we have oriented the faces of $P$ so that we have incidence numbers $[F: G] \in\{-1,0,1\}$ at our disposal.

Definition 2.2.1 1. Given a diagram $A: F(P)_{0} \longrightarrow R$-Mod we define its ČECH complex to be the bounded chain complex $\check{\Gamma}_{P}(A)=\check{\Gamma}(A)$ given by

$$
\check{\Gamma}(A)_{s}:=\bigoplus_{\operatorname{dim} F=n-s} A^{F}
$$

the sum extending over all non-empty faces of $P$, with differentials given by $A^{G} \stackrel{[F: G]}{\longleftarrow} A^{F}$ for the pair $F \subseteq G$ of non-empty faces of $P$.

2. Let $Y: F(P)_{0} \longrightarrow \mathrm{Ch}(R)$ be a diagram of chain complexes of $R$-modules. We define the ČECH complex $\check{\Gamma}_{P}(Y)=\check{\Gamma}(Y)$ of $Y$ to be the total complex of the double chain complex of $R$-modules

$$
D_{s, t}(Y)=D_{s, t}=\bigoplus_{\operatorname{dim} F=n-s} Y_{t}^{F}
$$

with horizontal differentials given by $Y_{t}^{G} \stackrel{[F: G]}{\longleftarrow} Y_{t}^{F}$ and vertical differential given by the differential in $Y$ multiplied by the sign $(-1)^{S}$, cf. $[13,1.2 .5]$.

3. Any presheaf $Y \in \operatorname{Pre}(P)$ can be considered as a diagram of chain complexes of $R$-modules, and we define its ČECH complex $\check{\Gamma}_{P}(Y)=\check{\Gamma}(Y)$ as in (2).

Remark 2.2.2 1. The homology modules of $\check{\Gamma}(A)$ in 2.2 .1 (1) are isomorphic to higher derived inverse limits of the diagram $A$; more precisely, $\lim ^{k}(A) \cong$ $H_{n-k} \check{\Gamma}(A)$. See [4, 2.19] for a proof.

2. If $A: F(P)_{0} \longrightarrow R$-Mod is a constant diagram with value $A^{F}=M$ for all $F$, then $H_{n} \check{\Gamma}(A)=M$ and $H_{k} \check{\Gamma}(A)=0$ for $k \neq n$. This follows easily from (1), or from the observation that $\check{\Gamma}(A)$ is dual to the chain complex computing cellular homology of the polytope $P$ with coefficients in $M$, up to re-indexing.

3. If $R$ is a commutative ring and $Y \in \mathrm{qCoh}(P)$ is concentrated in chain degree 0 , then $Y$ determines a quasi-coherent sheaf $\mathcal{F}$ on the scheme $X_{P}$. By $[4,2.18]$ we have isomorphisms $H_{n-k} \check{\Gamma}(Y) \cong H^{k}\left(X_{P}, \mathcal{F}\right)$, the $k$ th cohomology module of $X_{P}$ with coefficients in the sheaf $\mathcal{F}$.

4. For the diagram $Y$ in 2.2 .1 (2) $D_{*, *}$ is concentrated in the first $n+1$ columns so that $D_{p, *}=0$ if $p<0$ or $p>n$. We have $D_{*, t}=\check{\Gamma}\left(Y_{t}\right)$ which is a chain complex computing $\lim ^{n-*} Y_{t}$. The double chain complex $D_{*, *}(Y)$ thus gives rise to a convergent (homological) spectral sequence

$$
E_{s, t}^{1}(Y)=\lim _{\leftarrow}^{n-s}\left(Y_{t}\right) \Longrightarrow H_{s+t} \check{\Gamma}(Y),
$$

cf. Proposition 2.1.1 (1). 
Remark 2.2.3 There is another standard spectral sequence which we will have occasion to use. Let $Y \in \operatorname{Pre}(P)$, or more generally, let $Y$ be a diagram $F(P)_{0} \longrightarrow \mathrm{Ch}(R)$. Denote by $D_{*, *}$ the double complex of $R$-modules associated to $Y$ (2.2.1.1). Filtration by columns yields a convergent $E^{1}$-spectral sequence

$$
E_{p, q}^{1}(Y)=\bigoplus_{\operatorname{dim} F=n-p} H_{q}\left(Y^{F}\right) \Longrightarrow H_{p+q} \check{\Gamma}(Y)
$$

cf. [13, 5.6.1]; by Remark 2.2.2(1), $E_{p, q}^{2}(Y)=\lim _{\leftarrow}^{n-p} H_{q}(Y)$.

Proposition 2.2.4 Formation of ČECH complexes is homotopy invariant. More precisely, let $f: Y \longrightarrow Z$ be a map of $F(P)_{0}$-diagrams of $R$-module chain complexes. Suppose that for each $F \in F(P)_{0}$ the $F$-component of $f$ is a quasi-isomorphism. Then $f$ induces a quasi-isomorphism $\check{\Gamma}(Y) \longrightarrow \check{\Gamma}(Z)$.

Proof Consider the spectral sequence 2.2.3 for $Y$ and $Z$. By hypothesis, the map $f$ induces an isomorphism of $E^{1}$-spectral sequences $E_{*, *}^{1}(Y) \cong E_{*, *}^{1}(Z)$, hence induces an isomorphism of their abutments. But this is just a reformulation of the claim.

\subsection{Line bundles determined by $P$}

The polytope $P$ determines a family of objects of $\mathrm{qCoh}(P)$ as follows. For $k \in \mathbb{Z}$ we define

$$
\mathcal{O}(k): F \mapsto \mathcal{O}(k)^{F}=R\left[\left(k F+T_{F}\right) \cap \mathbb{Z}^{n}\right],
$$

considered as a diagram of chain complexes concentrated in degree 0 . Here $T_{F}$ is the tangent cone of $P$ at $F$, and $k F+T_{F}=\left\{k f+v \mid f \in F, v \in T_{F}\right\}$ is the MinKowSKI sum of the dilation $k F$ of $F$ and the cone $T_{F}$. The symbol $R[S]$ means the free $R$-module with basis $S$.

It is not difficult to see that $\mathcal{O}(k)$ is an object of qCoh $(P)$; the $A_{F}$-module structure of $\mathcal{O}(k)^{F}$ is induced by the translation action of the monoid $T_{F} \cap \mathbb{Z}^{n}$ on the set of integral points in $k F+T_{F}$. In fact, $\mathcal{O}(k)^{F}$ is a free $A_{F}$-module of rank 1 .

Proposition 2.3.1 For $k \in \mathbb{Z}$ and $j \in \mathbb{N}$ there is an isomorphism

$$
H_{j} \check{\Gamma}(\mathcal{O}(k)) \cong \begin{cases}R\left[k P \cap \mathbb{Z}^{n}\right] & \text { if } j=n \text { and } k \geqslant 0, \\ R\left[(\text { int } k P) \cap \mathbb{Z}^{n}\right] & \text { if } j=0 \text { and } k<0, \\ 0 & \text { otherwise. }\end{cases}
$$

Proof For $R=\mathbb{C}$ this is the standard calculation of cohomology of the line bundles $\mathcal{O}(k)$ on the toric variety $X_{P}$. Details are contained, for example, in $[5,2.5 .3]$; the calculation given there remains valid for arbitrary rings with unit.

It follows from Theorem 1.4.1 that the number $n_{P}$ defined in Corollary 1.4.2 can be characterised as being minimal among those $k \geqslant 0$ for which $\mathcal{O}(-k-1)$ is not acyclic (i.e., $\check{\Gamma} \mathcal{O}(-k-1)$ is not acyclic). 


\subsection{Twisting sheaves}

Definition 2.4.1 Let $Y \in$ Pre $(P)$ and $k \in \mathbb{Z}$. We define the $k$ th $t$ wist of $Y$, denoted $Y(k)$, as the objectwise tensor product of $\mathcal{O}(k)$ and $Y$. Explicitly,

$$
Y(k): F \mapsto Y(k)^{F}=\mathcal{O}(k)^{F} \otimes_{A_{F}} Y^{F}
$$

with structure maps induced by those of $\mathcal{O}(k)$ and $Y$.

By definition $Y(k)^{F}$ is isomorphic to the tensor product of $Y^{F}$ with a free $A_{F}$-module of rank 1, hence $Y(k)^{F}$ is non-canonically isomorphic to $Y^{F}$. The following properties are easily verified:

Lemma 2.4.2 1. For $Y \in \operatorname{Pre}(P)$ and $k, \ell \in \mathbb{Z}$ there is an isomorphism $Y(k)(\ell) \cong$ $Y(k+\ell)$. Moreover $Y(0) \cong Y$, this last isomorphism being natural in $Y$.

2. If $Y \in \mathrm{qCoh}(P)$ then $Y(k) \in \mathrm{qCoh}(P)$ for every $k \in \mathbb{Z}$.

3. If $Y \in \mathrm{hCoh}(P)$ then $Y(k) \in \mathrm{hCoh}(P)$ for every $k \in \mathbb{Z}$.

4. If $Y \in \operatorname{Perf}(P)$ then $Y(k) \in \operatorname{Perf}(P)$ for every $k \in \mathbb{Z}$.

\subsection{Quasi-coherent functors}

Definition 2.5.1 A quasi-coherent functor $\mathrm{Y}$ is an object $Y \in \mathrm{qCoh}(P)$ which is concentrated in chain degree 0 .

Lemma 2.5.2 Let $Y$ be a quasi-coherent functor, let $F \in F(P)_{0}$, and let $s_{F} \in Y^{F}$. Then there exists $k \in \mathbb{Z}$ and a map $f: \mathcal{O}(k) \longrightarrow Y$ such that $s_{F}$ is in the image of the $F$-component of $f$.

Proof The proof is a translation of the corresponding algebro-geometric fact [3, II.5.14 (b)] into combinatorial language. To begin with, we may assume that each face of $P$ has a lattice point in its relative interior. Indeed, we can replace $P$ by its dilate $(n+1) P$; note that this does not change the poset $F(P)_{0}$, nor does it change the cones, monoids and algebras constructed from $P$. Twisting translates easily: the sheaf $\mathcal{O}(1)$ computed with respect to $(n+1) P$ is precisely the sheaf $\mathcal{O}(n+1)$ computed with respect to $P$.

All algebras and modules constructed from $P$ are $R$-submodules of the free $R$-module $R\left[\mathbb{Z}^{n}\right]$. An element $v_{F} \in \mathbb{Z}^{n}$ gives rise to an element $x_{F}=1 \cdot v_{F} \in R\left[\mathbb{Z}^{n}\right]$. We think of the $x$-symbols as multiplicative, that is, we write $x_{F} / x_{G}$ for the module element associated to the vector $v_{F}-v_{G}$.

Now choose, for each $F \in F(P)_{0}$, a lattice point $v_{F}$ in the relative interior of $F$. It is not difficult to see that $\mathcal{O}(N)^{G}=R\left[\left(N G+T_{G}\right) \cap \mathbb{Z}^{n}\right]$, considered as a free $R$-module, has basis given by $N v_{G}+S_{G} \subseteq \mathbb{Z}^{n}$ so that

$$
\mathcal{O}(N)^{G}=x_{G}^{N} A_{G}, \quad N \in \mathbb{Z} .
$$

Moreover, if $p \in \mathcal{O}(N)^{G}$ then $x_{F}^{M} p \in \mathcal{O}(M+N)^{G}$ for all $M \geqslant 0$. In fact, $x_{F}^{M} p$ is the image of $x_{F}^{M} \otimes p$ under the isomorphism $\mathcal{O}(M) \otimes \mathcal{O}(N) \cong \mathcal{O}(M+N)$ restricted to $G$-components. 
As notational convention, given an element $x \in Z^{G}$ for a quasi-coherent functor $Z$ we call the image $\left.x\right|_{H}$ of $x$ under the structure map $Z^{G} \longrightarrow Z^{H}$ the restriction of $x$ to $H$.

Let us now start with the actual proof. By definition of tangent cones $v_{F}-v_{G} \in T_{G}$ for each $G \in F(P)_{0}$ so that $x_{F} / x_{G} \in A_{G}$. One can show the following:

2.5.2.1 For each $G \in F(P)_{0}$ we have

$$
T_{F \vee G}=T_{G}+\mathbb{R}\left(v_{F}-v_{G}\right) \quad \text { and } \quad S_{F \vee G}=S_{G}+\mathbb{Z}\left(v_{F}-v_{G}\right)
$$

where $F \vee G$ is the join of $F$ and $G$, that is, the smallest face of $P$ containing $F \cup G$. Consequently, the algebra $A_{F \vee G}$ is obtained from $A_{G}$ by localising by the single element $x_{F} / x_{G}$ in the centre $Z\left(A_{G}\right)$ of $A_{G}$.

This implies that for a large enough positive integer $N$ the element $s_{G}=$ $\left.\left(x_{F} / x_{G}\right)^{N} \cdot s_{F}\right|_{F \vee G}$ is in $Y^{F \vee G} \cong A_{F \vee G} \otimes_{A_{G}} Y^{G}$, where $s_{F}$ is the element given in the formulation of the Lemma. We may pick an integer $N$ which works for all $G \in F(P)_{0}$ simultaneously. Then for all $G, x_{G}^{N} \otimes s_{G}$ is an element of $Y(N)^{G}=(\mathcal{O}(N) \otimes Y)^{G}$ which restricts to $\left.x_{F}^{N} \otimes s_{F}\right|_{F \vee G} \in(\mathcal{O}(N) \otimes Y)^{F \vee G}$ (note here that $\left.\left.\left(x_{F}^{N} \otimes s_{F}\right)\right|_{F \vee G}=x_{F}^{N} \otimes\left(\left.s_{F}\right|_{F \vee G}\right)\right)$.

Now let $G \subseteq H \in F(P)_{0}$ be an arbitrary pair of non-empty faces of $P$. We do not know whether the elements $\left.x_{G}^{N} \otimes s_{G}\right|_{H}$ and $x_{H}^{N} \otimes s_{H}$ agree, but we know that after restricting further to $F \vee H$ both agree with $\left.x_{F}^{N} \otimes s_{F}\right|_{F \vee H}$. Consequently, using 2.5.2.1 again, for all large integers $M$ we have equality

$$
\left.\left(\frac{x_{F}}{x_{H}}\right)^{M} \cdot x_{G}^{N} \otimes s_{G}\right|_{H}=\left(\frac{x_{F}}{x_{H}}\right)^{M} \cdot x_{H}^{N} \otimes s_{H} \in Y(N)^{H} .
$$

Now multiplication with $x_{H}^{M}$ yields an isomorphism $Y(N)^{H} \cong Y(M+N)^{H}$ so that the above equality becomes

$$
\left.\left(x_{F}^{M} x_{G}^{N}\right) \otimes s_{G}\right|_{H}=\left(x_{F}^{M} x_{H}^{N}\right) \otimes s_{H} \in Y(M+N)^{H},
$$

with both elements restricting to $\left.x_{F}^{M+N} \otimes s_{F}\right|_{F \vee H}$ on $F \vee H$.

To sum up, we have shown that the family of elements $\left(x_{F}^{M} x_{G}^{N}\right) \otimes s_{G}, G \in F(P)_{0}$, determines an element $e$ in $\lim _{\leftarrow} Y(M+N)$, and hence an $R$-module homomorphism $R \longrightarrow \lim _{\leftarrow} Y(M+N)$ which sends $1 \in R$ to $e$. But then, by forcing equivariance, there is a map $\mathcal{O}(0) \longrightarrow Y(M+N)$ which sends $1 \in A_{G}$ to $\left(x_{F}^{M} x_{G}^{N}\right) \otimes s_{G}$. Twisting by $-(M+N)$ yields a map $\mathcal{O}(-M-N) \longrightarrow Y$ such that $s_{G}$ is in the image of the $G$-component. This applies in particular to $G=F$ which is the case of the Lemma.

Corollary 2.5.3 Let $Y$ be a quasi-coherent functor such that for all $F \in F(P)_{0}$ the $A_{F}$-module $Y^{F}$ is finitely generated. Then there are finitely many numbers $n_{i} \in \mathbb{Z}$ and a map

$$
\bigoplus_{i} \mathcal{O}\left(n_{i}\right) \longrightarrow Y
$$

which is surjective on each component. 
Proof For $F \in F(P)_{0}$ choose generators $s_{1}^{F}, \ldots, s_{\ell(F)}^{F}$. By Lemma 2.5.2 there are maps $f_{i}^{F}: \mathcal{O}\left(n_{i}^{F}\right) \longrightarrow Y$ such that $s_{i}^{F}$ is in the image of $f_{i}^{F}$. The required map is given by the sum

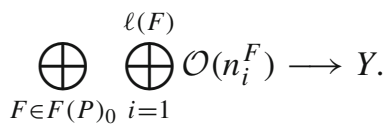

Lemma 2.5.4 Let $R$ be a left NOETHERian ring. Let $Y$ be a quasi-coherent functor such that for all $F \in F(P)_{0}$ the $A_{F}$-module $Y^{F}$ is finitely generated. Then $H_{k} \check{\Gamma}(Y)$ is trivial for $k<0$ and $k>n$, and is a finitely generated $R$-module for all $k \in \mathbb{Z}$.

Proof This follows the pattern of [3, III.5.2]. Triviality of $H_{k} \check{\Gamma}(Y)$ for $k<0$ and $k>n$ is immediate as $\check{\Gamma}(Y)$ is concentrated in degrees 0 to $n$, by construction.

The Lemma is true for a finite sum of quasi-coherent functors of the form $\mathcal{O}(k)$, by the calculation in Proposition 2.3.1. By Corollary 2.5.3 we can find a surjection $Z \longrightarrow Y$ with $Z$ a finite sum of $\mathcal{O}(k)$ s. Let $K$ denote the kernel; this is a quasicoherent functor as well. By construction we obtain a short exact sequence of chain complexes

$$
0 \longrightarrow \check{\Gamma} K \longrightarrow \check{\Gamma} Z \longrightarrow \check{\Gamma} Y \longrightarrow 0
$$

Now use increasing induction on $k$ on the corresponding exact sequence snippet

$$
H_{k+1} \check{\Gamma} Z \longrightarrow H_{k+1} \check{\Gamma} Y \longrightarrow H_{k} \check{\Gamma} K
$$

starting with $k=-1$; by choice of $Z$ the module on the left is finitely generated, and by what has been established by induction the module on the right is finitely generated as well. Since $R$ is left NOETHERian it follows that the middle module is finitely generated.

Proposition 2.5.5 Suppose $R$ is left NOETHERian. Let $Y \in \mathrm{hCoh}(P)$ be such that $Y_{k}^{F}$ is finitely generated as an $A^{F}$-module for all $F \in F(P)_{0}$ and all $k \in \mathbb{Z}$. Then $\check{\Gamma}(Y)$ is a (possibly unbounded) chain complex with finitely generated homology modules.

Proof Since $Y$ is a homotopy sheaf $H_{q}(Y): F \mapsto H_{q}\left(Y^{F}\right)$ is a quasi-coherent functor (this uses Lemma 1.1.2 and the fact that taking homology is compatible with localisation). Since $R$ is left NOETHERian, and since all the modules $Y_{k}^{F}$ are finitely generated, the modules $H_{q}\left(Y^{F}\right)$ are finitely generated as well. It follows from Lemma 2.5.4, applied to $H_{q}(Y)$, and from Remark 2.2 .2 (1) that all the entries of the $E^{2}$-term of the spectral sequence 2.2.3 are finitely generated $R$-modules. Since this spectral sequence is concentrated in columns 0 to $n$, its abutment $H_{p+q} \check{\Gamma} Y$ consists of finitely generated $R$-modules as well (making use the fact that $R$ is left NOETHERian once again). 


\subsection{Finiteness of the ČECH complex}

We are now going to prove the following fundamental finiteness result:

Theorem 2.6.1 Let $R$ be a unital ring. Suppose that $R$ is commutative, or else left NOETHERian. Let $Y \in \operatorname{Perf}(P)$. Then $\check{\Gamma}(Y)$ is a perfect complex of $R$-modules.

Proof Suppose first that $R$ is left NOETHERian. Since $Y$ is a perfect complex, there exists a homotopy vector bundle $V \in$ hVect $(P)$ together with a quasi-isomorphism $V \longrightarrow Y$ by Lemma 1.1.7. Then the induced map $\check{\Gamma} V \longrightarrow \check{\Gamma} Y$ is a quasi-isomorphism by Proposition 2.2.4. Since $V$ is bounded so is $\check{\Gamma} V$. Since $V^{F}$ consists of projective $A_{F}$-modules and since $A_{F}$ is free as an $R$-module, $\check{\Gamma} V$ consists of projective $R$-modules, and has finitely generated homology modules by Proposition 2.5.5. But this means that $\check{\Gamma} V$ is chain homotopy equivalent to a strict perfect complex of $R$-modules [9, 1.7.13].

Now suppose that $R$ is commutative, but not NOETHERian. Then $Y$ can be replaced, up to quasi-isomorphism, by a bounded complex $V$ in hVect $(P) \cap \mathrm{qCoh}(P)$; this is true since the toric scheme $X_{P}$ is projective over Spec $R$ and thus has an ample line bundle, so we can appeal to Lemma 1.3 .1 and $[11,2.3 .1$ (d)]. Then $\check{\Gamma}(Y) \simeq \check{\Gamma}(V)$, and it is enough to prove the Theorem for $V$ only.

The complex $V$ descends to a NOETHERian subring $R_{0}$. More precisely, write qCoh $(P)_{0}$ for the category qCoh $(P)$ defined over a subring $R_{0}$ instead of $R$, and similarly for hVect $(P)_{0}$. Then by NOETHERian approximation [11, Appendix $\mathrm{C}$ ], there is a NOETHERian subring $R_{0}$ of $R$, and a bounded complex $V_{0} \in \mathrm{hVect}(P)_{0} \cap \mathrm{qCoh}(P)_{0}$ such that $V=R \otimes_{R_{0}} V_{0}$. By the first part of the proof there is a strict perfect complex $B_{0}$ of $R_{0}$-modules which is chain homotopy equivalent to $\check{\Gamma} V_{0}$. But then $\check{\Gamma} V \cong R \otimes_{R_{0}} \check{\Gamma} V_{0}$ is homotopy equivalent to $R \otimes_{R_{0}} B_{0}$, and the latter is strict perfect.

\subsection{Canonical sheaves and suspension of chain complexes}

Definition 2.7.1 1. For $k \in \mathbb{Z}$ and $C \in \mathrm{Ch}(R)$ we define $\mathcal{O}(k) \otimes C$ to be the sheaf given by

$$
(\mathcal{O}(k) \otimes C)^{F}=\mathcal{O}(k)^{F} \otimes_{R} C \text { for } F \in F(P)_{0}
$$

with structure maps induced by those of $\mathcal{O}(k)$. We call $\mathcal{O}(k) \otimes C$ the $k$ th canonical sheaf associated to $C$.

2. Let $C$ be an $R$-module chain complex. We denote by con $(C)$ the constant $F(P)_{0^{-}}$ diagram with value $C$ and identity structure maps.

3. The $n$th suspension $C[n]$ of the chain complex $C \in \mathrm{Ch}(R)$ is defined by $C[n]_{k}=$ $C_{k-n}$, and multiplying the differentials with the sign $(-1)^{n}$.

Lemma 2.7.2 Let $C \in \mathrm{Ch}(R)$. If $k<0$ is an integer such that $E_{P}(k)=0$ (i.e., such that $k P$ has no lattice points in its interior $)$, then $\Gamma(\mathcal{O}(k) \otimes C)$ is acyclic.

Proof Let $D_{*, *}=D_{*, *}(\mathcal{O}(k) \otimes C)$ be the double chain complex associated to $\mathcal{O}(k) \otimes$ $C$, cf. (2.2.1.1). The $A_{F}$-module $\mathcal{O}(k)^{F} \cong A_{F}$ is a free $R$-module for each $F \in F(P)_{0}$, 
and the $\check{C}$ ECH complex $\check{\Gamma} \mathcal{O}(k)$ has the property that the image of each chain module under the differential is a free $R$-module (since all maps are given by inclusion of bases). Hence by the KUNNETH formula $[13,3.6 .1]$ the homology of the horizontal chain complex $D_{*, p}$ fits into a short exact sequence

$$
H_{k}(\check{\Gamma} \mathcal{O}(k)) \otimes_{R} C_{p} \longrightarrow H_{k}\left(\check{\Gamma} \mathcal{O}(k) \otimes_{R} C_{p}\right) \longrightarrow \operatorname{Tor}_{1}^{R}\left(H_{n-1} \check{\Gamma} \mathcal{O}(k), C_{p}\right)
$$

By hypothesis $E_{P}(k)=0$ so that $\check{\Gamma} \mathcal{O}(k)$ is acyclic by Proposition 2.3.1. So first and third term of the short exact sequence are trivial, hence so is the middle term.

But this means that the spectral sequence (2.2.2.1) associated to $D_{*, *}$ has trivial $E^{1}$-term, hence its abutment $H_{*} \check{\Gamma}(\mathcal{O}(k) \otimes C)$ is trivial too. This proves the Lemma.

Lemma 2.7.3 For every chain complex $C \in \mathrm{Ch}(R)$ we have a canonical quasi-isomorphism $C[n] \stackrel{\simeq}{\rightarrow} \check{\Gamma} \operatorname{con}(C)$.

Proof Let $A_{*, *}$ denote the complex $C$ considered as a double chain complex concentrated in column $n$. That is, we're looking at the double chain complex with $A_{n, k}=$ $C_{k}, A_{j, k}=0$ for $j \neq n$, and vertical differential the differential of $C$ multiplied with the sign $(-1)^{n}$. Then the total complex of $A_{*, *}$ is precisely $C[n]$.

The double chain complex $A_{*, *}$ maps into the double chain complex $D_{*, *}=$ $D_{*, *}(\operatorname{con}(C))$ associated to con $(C)$ by the diagonal map $\Delta$ given by

$$
C_{t} \longrightarrow \bigoplus_{\operatorname{dim} F=n} C_{t}=D_{n, t}, t \in \mathbb{Z}
$$

This defines indeed a map of double chain complexes by the properties of incidence numbers; more precisely, the horizontal chain complex $D_{*, t}$ is the tensor product of $C_{t}$ with the dual of the chain complex computing the integral cellular homology of $P$, and the map of horizontal chain complexes $A_{*, t} \longrightarrow D_{*, t}$ is the tensor product of $C_{t}$ with the dual of the augmentation map. In particular, the map is a quasi-isomorphism with respect to "horizontal" homology. By the spectral sequence argument 2.1.1 (2), $\Delta$ induces a quasi-isomorphism

$$
C[n]=\operatorname{Tot} A_{*, *} \stackrel{\operatorname{Tot} \Delta}{\longrightarrow} \operatorname{Tot} D_{*, *}=\check{\Gamma}(\operatorname{con}(C)) .
$$

Lemma 2.7.4 For every chain complex $C \in \mathrm{Ch}(R)$ we have a canonical map con $(C) \longrightarrow \mathcal{O}(0) \otimes C$, induced by the inclusions of $F$-components

$$
\operatorname{con}(C)^{F}=C \cong R[\{0\}] \otimes_{R} C \longrightarrow R\left[\mathbb{Z}^{n} \cap C_{F}\right] \otimes_{R} C=(\mathcal{O}(0) \otimes C)^{F},
$$

which in turn induces a quasi-isomorphism $\check{\Gamma} \operatorname{con}(C) \stackrel{\simeq}{\rightarrow} \check{\Gamma}(\mathcal{O}(0) \otimes C)$. 
Proof First recall that $\mathcal{O}(0)^{F}=A_{F}=R\left[S_{F}\right]$ for each $F \in F(P)_{0}$; this means that we can consider $\mathcal{O}(0)^{F}$ as a $\mathbb{Z}^{n}$-graded $R$-module with homogeneous components 0 or $R$. The double chain complex $D_{*, *}(\mathcal{O}(0) \otimes C)$ associated to $\mathcal{O}(0) \otimes C$ is a double chain complex of $\mathbb{Z}^{n}$-graded $R$-modules with horizontal and vertical differentials respecting the grading. We can thus concentrate on homogeneous components one at a time. As explained in $[5,2.5 .3]$, the component of degree $0 \in \mathbb{Z}^{n}$ of the horizontal chain complex $D_{*, t}(\mathcal{O}(0) \otimes C)$ is the tensor product of $C_{t}$ with the dual of the chain complex calculating the integral cellular homology of $P$ so that its horizontal homology is concentrated in column $n$ and has value $C_{t}$. For all homogeneous degrees different from 0 the chain complex is acyclic (loc. cit.).

On the other hand, we have a canonical isomorphism of diagrams

$$
\operatorname{con}(C) \cong \operatorname{con}(R) \otimes C
$$

where con $(R)$ is the constant diagram with value $R$, considered as a diagram of chain complexes concentrated in chain degree 0 . We can think of $R$ as a $\mathbb{Z}^{n}$-graded module concentrated in degree $0 \in \mathbb{Z}^{n}$. Thus the associated double chain complex $D_{*, *}(\operatorname{con}(R) \otimes C)$ consists of $\mathbb{Z}^{n}$-graded modules with differentials preserving the grading. In homogeneous degree 0 , the horizontal chain complexes $D_{*, t}(\operatorname{con}(R) \otimes C)$ and $D_{*, t}(\mathcal{O}(0) \otimes C)$ agree, in non-zero degrees the horizontal chain complex $D_{*, t}(\operatorname{con}(R) \otimes C)$ is the zero-complex.

We have an obvious map of double complexes

$$
\omega: D_{*, *}(\operatorname{con}(R) \otimes C) \longrightarrow D_{*, *}(\mathcal{O}(0) \otimes C),
$$

the inclusion of degree 0 components, which by the previous two paragraphs induces an isomorphism on horizontal homology modules. By the spectral sequence argument 2.1.1 (2) the composite

$$
\begin{aligned}
\check{\Gamma} \operatorname{con}(C) & \cong \check{\Gamma}(\operatorname{con}(R) \otimes C)=\operatorname{Tot} D_{*, *}(\operatorname{con}(R) \otimes C) \\
& \underset{\omega}{\simeq} \operatorname{Tot} D_{*, *}(\mathcal{O}(0) \otimes C)=\check{\Gamma}(\mathcal{O}(0) \otimes C)
\end{aligned}
$$

is thus a quasi-isomorphism.

\subsection{A model for suspension}

For any presheaf $Y \in \operatorname{Pre}(P)$ we let $Y[n]$ denote the $n$th suspension of $Y$, that is, the diagram given by $F \mapsto Y[n]^{F}=Y^{F}[n]$, cf. Definition 2.7.1 (3), with structure maps induced by those of $Y$.

Definition 2.8.1 Let $F$ be a face of $P$ (possibly empty), and let $Y \in$ Pre $(P)$. We define a new presheaf $F_{*} Y$ by the rule

$$
F_{*} Y: G \mapsto Y^{G \vee F}
$$


where $G \vee F$ is the join of $G$ and $F$, that is, the smallest face of $P$ containing $G \cup F$, and $\left(F_{*} Y\right)^{G}=Y^{F \vee G}$ is considered as an $A_{G}$-module chain complex by restriction of scalars.

Remark 2.8.2 If $R$ is a commutative ring and $Y \in \mathrm{qCoh}(P)$ is a sheaf, let $\mathcal{F}$ denote the chain complex of quasi-coherent sheaves on $X_{P}$ determined by $Y$. Then $F_{*} Y$ corresponds to $j_{*}\left(\left.\mathcal{F}\right|_{U_{F}}\right)=j_{*}\left(\widetilde{Y^{F}}\right)$ where $j: U_{F} \longrightarrow X_{P}$ is the inclusion.

The construction of $F_{*} Y$ is natural in $Y$ : For $F \subseteq F^{\prime}$ a pair of faces of $P$, the structure maps of $Y$ induce a map of presheaves $F_{*} Y \longrightarrow F_{*}^{\prime} Y$. Moreover, every entry of $F_{*}(Y)$ is an $A_{F}$-module, by restriction of scalars $\left(A_{\emptyset}=R\right.$ here) so that $\check{\Gamma} F_{*} Y$ is a chain complex of $A_{F}$-modules. Hence the following definition is meaningful:

Definition 2.8.3 For $Y \in \operatorname{Pre}(P)$ we define a new presheaf $\sigma Y$ by

$$
F \mapsto(\sigma Y)^{F}=\check{\Gamma}\left(F_{*} Y\right) .
$$

Lemma 2.8.4 Let $Y \in$ Pre $(P)$. There is a natural map of presheaves

$$
\alpha: Y[n] \longrightarrow \sigma Y
$$

which is a quasi-isomorphism on each component. In particular, if $Y$ is a homotopy sheaf then so is $\sigma Y$, and if $Y$ is a perfect complex so is $\sigma Y$.

Proof Let $\gamma Y$ denote the presheaf $(\gamma Y)^{F}=\check{\Gamma} \operatorname{con}\left(Y^{F}\right)$. For any face $F \in F(P)_{0}$ the structure maps of $Y$ induce a map of diagrams con $\left(Y^{F}\right) \longrightarrow F_{*} Y$ and thus a map $\check{\Gamma} \operatorname{con}\left(Y^{F}\right) \longrightarrow \check{\Gamma} F_{*} Y$. This construction is natural in $F$ so we obtain maps of presheaves

$$
Y[n] \longrightarrow \gamma Y \longrightarrow \sigma Y
$$

the first one consisting of the canonical quasi-isomorphisms of Lemma 2.7.3. The composition of these two maps is the $\alpha$ of the Lemma, and we are left to prove that the map

$$
(\gamma Y)^{F}=\check{\Gamma} \operatorname{con}\left(Y^{F}\right) \longrightarrow \check{\Gamma} F_{*} Y=(\sigma Y)^{F}
$$

is a quasi-isomorphism for each $F \in F(P)_{0}$; we will use the (by now familiar) spectral sequence comparison argument.

Write st $F=\left\{G \in F(P)_{0} \mid G \supseteq F\right\}$, a sub-poset of $F(P)_{0}$. Given a diagram $A: F(P)_{0} \longrightarrow R$-Mod we can consider its restriction $\left.A\right|_{\text {st } F}$ to the poset st $F$. Conversely, a diagram $B:$ st $F \longrightarrow R$-Mod can be extended to a diagram

$$
F_{*} B: F(P)_{0} \longrightarrow R \text {-Mod }, \quad G \mapsto B^{G \vee F} .
$$

In fact, extension and restriction form an adjoint pair, with restriction being the left adjoint. Both functors are exact. 
Now let $B:$ st $F \longrightarrow R$-Mod be given, and let $B \longrightarrow I^{\bullet}$ be an injective resolution of $B$. Then $F_{*} B \longrightarrow F_{*} I^{\bullet}$ is an injective resolution of $F_{*} B$. Consequently, we have

$$
\begin{aligned}
{\underset{\leftarrow}{\lim }}^{q} B & =H^{q} \lim _{\text {st } F} I^{\bullet} \\
& =H^{q} \operatorname{hom}\left(\left.(\operatorname{con} R)\right|_{\mathrm{st} F}, I^{\bullet}\right) \\
& =H^{q} \operatorname{hom}\left(\operatorname{con} R, F_{*} I^{\bullet}\right) \\
& =H^{q} \lim F_{*} I^{\bullet} \\
& =\lim _{\leftarrow}^{q} F_{*} B .
\end{aligned}
$$

On the other hand, st $F$ has minimal element $F$ so that $\lim B=B^{F}$, and $\lim$ is exact; that is,

$$
\lim _{\leftarrow}^{q} B= \begin{cases}B^{F} & \text { if } q=0 \\ 0 & \text { else. }\end{cases}
$$

These calculations apply in particular to $B=\left.A\right|_{\text {st } F}$ for $A$ an $F(P)_{0}$-diagram of $R$-modules. Since $\left(F_{*}\left(\left.A\right|_{\mathrm{st} F}\right)\right)^{F}=A^{F}$ this means that

$$
\lim _{\leftarrow}^{q} F_{*}\left(\left.A\right|_{\text {st } F}\right)= \begin{cases}A^{F} & \text { if } q=0 \\ 0 & \text { else. }\end{cases}
$$

The calculations also imply that the obvious map con $\left.\left(B^{F}\right)\right|_{\mathrm{st} F} \longrightarrow B$, for arbitrary $B$ as before and its adjoint $\operatorname{con} B^{F} \longrightarrow F_{*} B$ induce isomorphisms

$$
\left.\lim _{\leftarrow} q\left(\operatorname{con} B^{F}\right)\right|_{\text {st } F} \cong \lim _{\leftarrow}^{q} B \text { and } \quad \lim _{\leftarrow} q \operatorname{con} B^{F} \cong \lim _{\leftarrow}^{q} F_{*} B
$$

as both are the identity on $F$-components.

Let us return to the map

$$
(\gamma Y)^{F}=\check{\Gamma} \operatorname{con}\left(Y^{F}\right) \longrightarrow \check{\Gamma} F_{*} Y=(\sigma Y)^{F} .
$$

It is induced by a map of double chain complexes

$$
D_{*, *}\left(\operatorname{con} Y^{F}\right) \longrightarrow D_{*, *}\left(F_{*} Y\right)
$$

which, when restricted to $t$ th horizontal chain complexes, is a map of chain complexes computing the higher derived limits $\lim ^{n-*}$ of the diagrams $A=\operatorname{con} Y_{t}^{F}$ and $F_{*}\left(\left.Y_{t}\right|_{\text {st } F}\right)$, respectively, by Remark $2.2 .2(\overleftarrow{1})$; by the calculation above, applied to $A$ and $B=\left.Y_{t}\right|_{\mathrm{st} F}$, this yields an isomorphism on homology modules for all values of $*$. In other words, the $E^{1}$-terms of the two spectral sequences are isomorphic, hence their abutments are as well, so the map in question is a quasi-isomorphism as claimed. 
2.9 Relating $\mathcal{O}(0) \otimes \check{\Gamma}$ to $n$th suspension

We proceed to construct a map between the functors $\mathcal{O}(0) \otimes \check{\Gamma}$ and $n$th suspension which will be used later in $K$-theoretical computations.

2.9.1 Let $Y \in \operatorname{Pre}(P)$, and fix $F \in F(P)_{0}$. The structure maps of $Y$ induce a canonical map $Y^{F} \longrightarrow \lim F_{*} Y$ and thus, by forcing equivariance, a map

$$
\rho^{F}: \mathcal{O}(0) \otimes Y^{F} \longrightarrow F_{*} Y
$$

Lemma 2.9.2 For $Y \in \operatorname{hCoh}(P)$ the $\operatorname{map} \check{\Gamma}\left(\rho^{F}\right): \check{\Gamma}\left(\mathcal{O}(0) \otimes Y^{F}\right) \longrightarrow \check{\Gamma} F_{*} Y$ is a quasi-isomorphism.

Proof The map $\check{\Gamma}\left(\rho^{F}\right)$ fits into a commutative square diagram

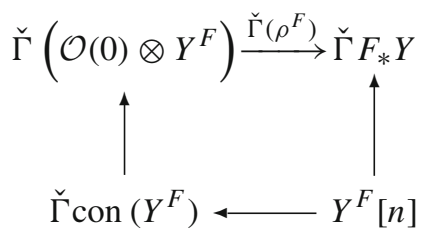

Left, bottom and right map are quasi-isomorphisms by Lemmas 2.7.4, 2.7.3 and 2.8.4, respectively, hence the top map is a quasi-isomorphism as well.

2.9.3 Let $Y \in \operatorname{Pre}(P)$. The structure maps of $Y$ induce, for each $F \in F(P)_{0}$, a map of presheaves $Y \longrightarrow F_{*} Y$ and hence a map of chain complexes $\check{\Gamma} Y \longrightarrow \check{\Gamma} F_{*} Y=$ $(\sigma Y)^{F}$. Since this is natural in $F$ we obtain a map

$$
\check{\Gamma} Y \longrightarrow \lim _{F \in F(P)_{0}} \check{\Gamma} F_{*} Y=\lim _{F \in F(P)_{0}}(\sigma Y)^{F}
$$

which, by forcing equivariance, defines a map

$$
\beta: \mathcal{O}(0) \otimes \check{\Gamma} Y \longrightarrow \sigma Y .
$$

Lemma 2.9.4 Let $Y \in \mathrm{hCoh}(P)$. Then $\check{\Gamma}(\beta): \check{\Gamma}(\mathcal{O}(0) \otimes \check{\Gamma} Y) \longrightarrow \check{\Gamma}(\sigma Y)$ is a quasi-isomorphism of chain complexes of $R$-modules.

Before we delve into the proof, we fix conventions regarding triple chain complexes. Suppose we have a threefold $R$-module chain complex $A_{*, *, *}$ : a $\mathbb{Z}^{3}$-indexed collection of $R$-modules $A_{x, y, z}$ together with pairwise commuting differentials

$$
\begin{aligned}
& \bar{\partial}_{x}: A_{x, y, z} \longrightarrow A_{x-1, y, z} \\
& \bar{\partial}_{y}: A_{x, y, z} \longrightarrow A_{x, y-1, z} \\
& \bar{\partial}_{z}: A_{x, y, z} \longrightarrow A_{x, y, z-1}
\end{aligned}
$$


which square to trivial maps. That is, we are looking at an object of the category $\mathrm{Ch}(\mathrm{Ch}(\mathrm{Ch}(R)))$. Then we can define new differentials by

$$
\partial_{x}=\bar{\partial}_{x}, \quad \partial_{y}=(-1)^{x} \bar{\partial}_{y}, \quad \partial_{z}=(-1)^{x+y} \bar{\partial}_{z}
$$

which are easily checked to anti-commute: $\partial_{i} \partial_{j}=\left(\delta_{i, j}-1\right) \partial_{j} \partial_{i}$ (where $\delta_{i, j}$ is the usual KRONECKER delta symbol). We say that the graded module $A_{*, *, *}$ together with the maps $\partial_{i}, i=x, y, z$ is a triple chain complex (in analogy to the established usage of the term double complex in the literature). We define the total complex $\operatorname{Tot}^{x, y, z}\left(A_{*, *, *}\right)$ by setting

$$
\operatorname{Tot}^{x, y, z}\left(A_{*, *, *}\right)_{n}=\bigoplus_{x+y+z=n} A_{x, y, z}
$$

equipped with the differential defined by $\partial=\partial_{x}+\partial_{y}+\partial_{z}$. This is an $R$-module chain complex, the relation $\partial^{2}=0$ is easily verified.

The relevant observation here is that one can do the totalisation in two steps. Define $\operatorname{Tot}^{y, z}\left(A_{*, *, *}\right)$ by

$$
\operatorname{Tot}^{y, z}\left(A_{*, *, *}\right)_{p, q}=\bigoplus_{y+z=q} A_{p, y, z}
$$

this is a double chain complex when equipped with "horizontal" differential $\partial^{h}=\partial_{x}$ and "vertical" differential $\partial^{v}=\partial_{y}+\partial_{z}$. It is a matter of tracing definitions to see that we have an equality of chain complexes

$$
\operatorname{Tot}\left(\operatorname{Tot}^{y, z}\left(A_{*, *, *}\right)\right)=\operatorname{Tot}^{x, y, z}\left(A_{*, *, *}\right) .
$$

Similarly, we can define a double chain complex $\operatorname{Tot}^{x, z}\left(A_{*, *, *}\right)$ by

$$
\operatorname{Tot}^{x, z}\left(A_{*, *, *}\right)_{p, q}=\bigoplus_{x+z=p} A_{x, q, z}
$$

equipped with "horizontal" differential $\partial^{h}=\partial_{x}+\partial_{z}$ and "vertical" differential $\partial^{v}=$ $\partial_{y}$. We then have an equality of chain complexes

$$
\operatorname{Tot}\left(\operatorname{Tot}^{x, z}\left(A_{*, *, *}\right)\right)=\operatorname{Tot}^{x, y, z}\left(A_{*, *, *}\right) .
$$

Proof of Lemma 2.9.4 The map $\check{\Gamma}(\beta)$ is a map of $R$-module chain complexes which can, in fact, be described by a map of triple chain complexes. In more detail, define

$$
A_{x, y, z}=\bigoplus_{\operatorname{dim} G=n-x \operatorname{dim} F=n-y} \mathcal{O}(0)^{G} \otimes_{R} Y_{z}^{F}
$$


equipped with differentials $\bar{\partial}_{z}$ given by the differential in $Y^{F}$, and differentials $\bar{\partial}_{x}$ and $\bar{\partial}_{y}$ determined by incidence numbers $\left[G_{1}: G_{2}\right]$ and $\left[F_{1}: F_{2}\right]$, respectively. Similarly, define

$$
B_{x, y, z}=\bigoplus_{\operatorname{dim}} \bigoplus_{G=n-x \operatorname{dim} F=n-y} Y_{z}^{G \vee F}
$$

equipped with differentials as above. Then both $A_{x, y, z}$ and $B_{x, y, z}$ are threefold chain complexes and thus determine, by modification of the differentials, triple chain complexes as explained above. The structure maps of $Y$ induce a map of triple chain complexes $\gamma: A_{x, y, z} \longrightarrow B_{x, y, z}$. A tedious but straightforward tracing of signs and direct sums involved shows:

(i) $D_{*, *}(\mathcal{O}(0) \otimes \check{\Gamma} Y)=\operatorname{Tot}^{y, z}\left(A_{*, *, *}\right)$,

(ii) $D_{*, *}(\sigma Y)=\operatorname{Tot}^{y, z}\left(B_{*, *, *}\right)$,

(iii) $D_{*, *}(\beta)=\operatorname{Tot}^{y, z}(\gamma)$.

Since Tot $\circ \operatorname{Tot}^{y, z}=\operatorname{Tot}^{x, y, z}$ this implies that

$$
\operatorname{Tot}^{x, y, z}(\gamma)=\check{\Gamma}(\beta)
$$

Let us now consider the double chain complex map $\operatorname{Tot}^{x, z}(\gamma)$. We claim that $\operatorname{Tot}^{x, z}(\gamma)$ induces a quasi-isomorphism on horizontal chain complexes. By the usual spectral sequence argument 2.1.1, this implies that $\operatorname{Tot}^{x, y, z}(\gamma)=\operatorname{Tot}\left(\operatorname{Tot}^{x, z}(\gamma)\right)$ is a quasi-isomorphism of $R$-module chain complexes, hence so is $\check{\Gamma}(\beta)$ in view of (2.9.4.1).

Fix an index $q \in \mathbb{Z}$. The $q$ th row of the source of $\operatorname{Tot}^{x, z}(\gamma)$ has $p$ th entry

$$
\bigoplus_{\operatorname{dim} F=n-q} \bigoplus_{x+z=p} \bigoplus_{\operatorname{dim}} \mathcal{O}(0)^{G} \otimes_{R} Y_{z}^{F}
$$

and is thus of the form

$$
\bigoplus_{\operatorname{dim} F=n-q} \check{\Gamma}\left(\mathcal{O}(0) \otimes Y^{F}\right)
$$

up to the (constant!) sign $(-1)^{q}$ in the differential of $Y^{F}$. In particular this is non-trivial only for $0 \leqslant q \leqslant n$.

The $q$ th row of the target of $\operatorname{Tot}^{x, z}(\gamma)$ has $p$ th entry

$$
\bigoplus \bigoplus_{\operatorname{dim} F=n-q} \bigoplus_{x+z=p} Y_{z}^{G \vee F}=\bigoplus_{\operatorname{dim}} \bigoplus_{G=n-x} \bigoplus_{\operatorname{dim} F=n-q} \bigoplus_{x+z=p}\left(F_{*} Y\right)_{z}^{G}
$$

and is thus of the form

$$
\bigoplus_{\operatorname{dim} F=n-q} \check{\Gamma} F_{*} Y
$$

up to the (constant!) sign $(-1)^{q}$ in the differential of $F_{*} Y$. In particular this is nontrivial only for $0 \leqslant q \leqslant n$. 
Thus in row $q$ the map $\operatorname{Tot}^{x, z}(\gamma)$ is, up to sign $(-1)^{q}$ in the differentials of source and target, the direct sum of the maps $\rho^{F}$ defined in (2.9.1.1) with $\operatorname{dim} F=n-q$. By Lemma 2.9.2 this means that $\operatorname{Tot}^{x, z}(\gamma)$ is a quasi-isomorphism of horizontal chain complexes as claimed.

\section{Splitting the $K$-theory}

Let $R$ be a ring with unit. For this entire section we assume that $R$ is commutative, or else left NOETHERian.

\subsection{Reduced $K$-theory}

Recall that an $R$-module chain complex $C$ is called perfect if it is quasi-isomorphic to a strict perfect complex, that is, a bounded complex $B$ of finitely generated projective $R$-modules; if this is the case, there will always be a quasi-isomorphism $B \longrightarrow C$. Write Perf $(R)$ for the category of perfect chain complexes of $R$-modules. We write $K(R)$ for the $K$-theory space of the complicial biWALDHAUSEN category Perf $(R)$ equipped with quasi-isomorphisms as weak equivalences, and the degreewise split monomorphisms with cokernel in Perf $(R)$ as cofibrations.

For an $n$-dimensional polytope $P \subset \mathbb{R}^{n}$ we have defined the category Perf $(P)$ of perfect complexes in 1.1.6; recall that an object of Perf $(P)$ is a diagram indexed by the face lattice of $P$ with values in perfect chain complexes of modules over different rings, subject to a gluing condition. The $K$-theory space of Perf $(P)$ is denoted by $K(P)$, cf. Sect. 1.2.

Let Perf $(P)^{[0]}$ denote the full subcategory of those $Y \in \operatorname{Perf}(P)$ such that $\check{\Gamma}(Y)$ is acyclic, cf. Definition 2.2.1 (3). This is a complicial biWALDHAUSEN category with the usual conventions. Its associated $K$-theory space is called the reduced $K$-theory of $P$ and denoted $\tilde{K}(P)$.

We call a map $f: Y \longrightarrow Z$ in Perf $(P)$ an $h_{[0]}$-equivalence if $\check{\Gamma}(f)$ is a quasi-isomorphism; with respect to these maps as weak equivalences, Perf $(P)$ is (yet another) complicial biWALDHAUSEN category. Note that every quasi-isomorphism in Perf $(P)$ is an $h_{[0]}$-equivalence as the functor $\check{\Gamma}$ preserves quasi-isomorphisms by Proposition 2.2.4.

We will need the functor

$$
\psi_{0}: \operatorname{Perf}(R) \longrightarrow \operatorname{Perf}(P), C \mapsto \mathcal{O}(0) \otimes C .
$$

It is easy to see that $\psi_{0}$ takes values in perfect complexes. Indeed, for $C \in \operatorname{Perf}(C)$ there is a strict perfect complex of $R$-modules $D$ which is quasi-isomorphic to $C$. Since $A_{F}$ is a free $R$-module, for each $F \in F(P)_{0}$, taking tensor product with $A_{F}$ over $R$ is exact. Consequently, $\psi_{0}(Y)^{F}$ is quasi-isomorphic to $A_{F} \otimes_{R} D$ which is a strict perfect complex of $A_{F}$-modules.

Proposition 3.1.1 There is a fibration sequence of $K$-theory spaces

$$
\tilde{K}(P) \longrightarrow K(P) \stackrel{\check{\Gamma}}{\rightarrow} K(R)
$$


which has a section up to homotopy and up to sign induced by the functor $\psi_{0}$. Hence there is a splitting up to homotopy

$$
\tilde{K}(P) \times K(R) \simeq K(P) .
$$

Proof By the Fibration Theorem [12, 1.6.4] the sequences of exact functors of biWALDHAUSEN categories

$$
\left(\operatorname{Perf}(P)^{[0]}, w\right) \stackrel{\subseteq}{\rightarrow}(\operatorname{Perf}(P), w) \longrightarrow\left(\operatorname{Perf}(P), h_{[0]}\right)
$$

where $w$ stands for quasi-isomorphisms as weak equivalences, induces a fibration sequence of $K$-theory spaces.

We have exact functors

$$
\operatorname{Perf}(R) \stackrel{\psi_{0}}{\longrightarrow}\left(\operatorname{Perf}(P), h_{[0]}\right) \text { and }\left(\operatorname{Perf}(P), h_{[0]}\right) \stackrel{\check{\Gamma}}{\rightarrow} \operatorname{Perf}(R),
$$

the latter being well defined by Theorem 2.6.1. By Lemmas 2.7.3 and 2.7.4 we have a natural weak equivalence of functors from the $n$th suspension $C \mapsto C[n]$ to the composition $\check{\Gamma} \circ \psi_{0}$. Since suspension induces a self homotopy equivalence on the $K$-theory space $K(R)$, the functor $\check{\Gamma}$ is surjective on homotopy groups.

By Lemmas 2.8.4 and 2.9.4 there is a chain of natural transformation of functors represented by

$$
Y[n] \longrightarrow \sigma Y \longleftarrow \mathcal{O}(0) \otimes \check{\Gamma}(Y)=\psi_{0} \circ \check{\Gamma}(Y)
$$

which is in fact a chain of $h_{[0]}$-equivalences of functors. Thus $\check{\Gamma}$ is injective on homotopy groups.

In total, we have shown that the functor $\check{\Gamma}$ induces a homotopy equivalence from the $K$-theory of the base of the fibration sequence (3.1.1.1) to $K(R)$. The resulting fibration sequence

$$
\tilde{K}(P) \longrightarrow K(P) \stackrel{\check{\Gamma}}{\rightarrow} K(R)
$$

has section up to homotopy and up to sign induced by $\psi_{0}$ (as the composition $\check{\Gamma} \circ \psi_{0}$ is weakly equivalent to $n$th suspension, just as argued above) which yields the desired splitting.

\subsection{Further splitting}

If $\check{\Gamma}(\mathcal{O}(-1))$ happens to be acyclic, we can split off a further copy of $K(R)$ from the reduced $K$-theory $\tilde{K}(P)$; acyclicity of $\check{\Gamma} \mathcal{O}(-j)$ for $j>1$ allows to iterate the procedure. The argument is virtually the same as in Sect. 3.1, but the functors involve additional twisting. We record the details. 
For a given integer $k \geqslant 0$, let Perf $(P)^{[k]}$ denote the full subcategory of those $Y \in \operatorname{Perf}(P)$ such that $\check{\Gamma} Y(j)$ is acyclic for $0 \leqslant j \leqslant k$. This is a complicial biWALDHAUSEN category with the usual conventions. Its associated $K$-theory space is denoted $K(P,[k])$; in particular, $K(P,[0])=\tilde{K}(P)$.

We call a map $f: Y \longrightarrow Z$ in Perf $(P)$ an $h_{[k]}$-equivalence if $\check{\Gamma} f(j)$ is a quasi-isomorphism for $0 \leqslant j \leqslant k$; with respect to these maps as weak equivalences, Perf $(P)$ is (yet another) complicial biWALDHAUSEN category. Note that every quasi-isomorphism in Perf $(P)$ is an $h_{[k]}$-equivalence as both twisting and the functor $\check{\Gamma}$ preserve quasi-isomorphisms.

We will need the functors

$$
\psi_{k}: \operatorname{Perf}(R) \longrightarrow \operatorname{Perf}(P), C \mapsto \mathcal{O}(k) \otimes C
$$

( $k \in \mathbb{Z}$ here). One can show that this functor takes indeed values in perfect complexes; the argument is as for $\psi_{0}$. As a matter of notation, let us also introduce the $k$ th twist functor

$$
\theta_{k}: \operatorname{Perf}(P) \longrightarrow \operatorname{Perf}(P), Y \mapsto Y(k)
$$

Recall that the polytope $P$ determines a polynomial $E_{P}(x)$ with rational coefficients such that $\left|E_{P}(-j)\right|$ is the number of integral points in the interior of $-j P$ for integers $j \geqslant 1$, cf. Theorem 1.4.1. It follows that if $E_{P}(-j)=0$ for some $j>1$, then $E_{P}(-\ell)=0$ for $0<\ell \leqslant j$. Let $n_{P}$ be the number of distinct integral roots of $E_{P}(x)$; then $n_{P} \in[0, n]$, and if $n_{P} \neq 0$ then $n_{P}$ is maximal among the negatives of integer roots of $E_{P}(x)$.

Proposition 3.2.1 For $1 \leqslant \ell \leqslant n_{P}$ there is a fibration sequence of $K$-theory spaces

$$
K(P,[\ell]) \longrightarrow K(P,[\ell-1]) \stackrel{\check{\Gamma} \circ \theta_{\ell}}{\longrightarrow} K(R)
$$

which has a section up to homotopy and up to sign induced by the functor $\psi_{-\ell}$. Consequently, we have a homotopy equivalence

$$
K(P,[\ell]) \times K(R) \simeq K(P,[\ell-1]) .
$$

Proof The sequence of biWALDHAUSEN categories

$$
\left(\operatorname{Perf}(P)^{[\ell]}, w\right) \stackrel{\subseteq}{\rightarrow}\left(\operatorname{Perf}(P)^{[\ell-1]}, w\right) \longrightarrow\left(\operatorname{Perf}(P)^{[\ell-1]}, h_{[\ell]}\right)
$$

induces a fibration sequence of $K$-theory spaces, by the Fibration Theorem [12, 1.6.4]. We have to prove that the $K$-theory of its base is homotopy equivalent, via $\check{\Gamma} \circ \theta_{\ell}$, to $K(R)$, where we have written $\theta_{\ell}$ for the $\ell$ th twist functor $Y \mapsto Y(\ell)$.

First note that $\psi_{-\ell}$ restricts to an exact functor

$$
\psi_{-\ell}: \operatorname{Perf}(R) \longrightarrow\left(\operatorname{Perf}(P)^{[\ell-1]}, h_{[\ell]}\right) .
$$


We have to check that $\check{\Gamma} \circ \theta_{j}$, when applied to a complex of the form $\psi_{-\ell}(C)$, produces an acyclic chain complex for $0 \leqslant j<\ell$. But for $j$ in this range we have $-n_{P} \leqslant-\ell+j<0$ so that $E_{P}(-\ell+j)=0$. It follows that

$$
\check{\Gamma} \circ \theta_{j} \circ \psi_{-\ell}(C) \cong \check{\Gamma} C(-\ell+j)
$$

is acyclic by Lemma 2.7.2.

Now the composition $\left(\check{\Gamma} \circ \theta_{\ell}\right) \circ \psi_{-\ell} \cong \check{\Gamma} \psi_{0}$ is weakly equivalent to the $n$th suspension endo-functor $C \mapsto C[n]$ of $\mathrm{Ch}(R)$, by Lemmas 2.7.3 and 2.7.4, hence $\check{\Gamma} \circ \theta_{\ell}$ induces a surjection on homotopy groups of $K$-theory spaces.

According to Lemmas 2.8.4 and 2.9.4 the composition $\psi_{0} \circ \check{\Gamma}$ is connected to the $n$th suspension functor by a chain of $h_{[0]}$-equivalences depicted

$$
Y[n] \stackrel{\simeq}{\rightarrow} \sigma Y \triangleleft \psi_{0} \circ \check{\Gamma}(Y)
$$

(where the first map is actually a weak equivalence). Replacing $Y$ by its $\ell$ th twist $Y(\ell)$ yields a chain of $h_{[0]}$-equivalences

$$
\theta_{\ell} Y[n] \stackrel{\simeq}{\rightarrow} \sigma Y(\ell) \longleftarrow \psi_{0} \circ \check{\Gamma} \circ \theta_{\ell}(Y)
$$

Twisting by $-\ell$ again leaves us with a chain of natural maps

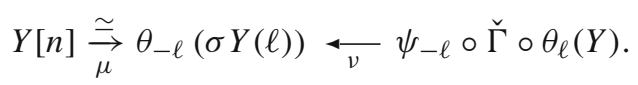

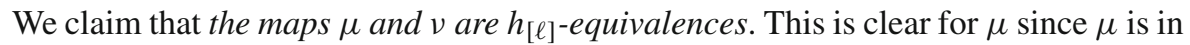
fact a weak equivalence. As for $\nu$, given an integer $j$ with $0 \leqslant j \leqslant \ell$ we have to prove that application of $\check{\Gamma} \circ \theta_{j}$ to the map $v$ produces a quasi-isomorphism of $R$-module chain complexes. For $0 \leqslant j<\ell$ this is true since both source and target of the resulting map of chain complexes are acyclic. Indeed, $\check{\Gamma} \circ \theta_{j}\left(\theta_{-\ell}(\sigma Y(\ell))\right) \simeq \check{\Gamma} Y(j)[n] \simeq 0$ since $Y$ is an object of Perf $(P)^{[\ell-1]}$, while $\check{\Gamma} \circ \theta_{j} \circ \psi_{-\ell} \circ \check{\Gamma} \circ \theta_{\ell}(Y)=\check{\Gamma} \circ \psi_{j-\ell} \circ \check{\Gamma} \circ \theta_{\ell}(Y) \simeq 0$ by Lemma 2.7.2, applied to the chain complex $C=\check{\Gamma} \circ \theta_{\ell}(Y)$, since $E_{P}(j-\ell)=0$. For $j=\ell$ note that $\theta_{\ell}(v)$ is the map $\sigma Y(\ell) \longleftarrow \psi_{0} \circ \check{\Gamma} Y(\ell)$ which is an $h_{[0]}$-equivalence according to Lemma 2.9.4 (applied to $Y(\ell)$ instead of $Y$ ).

We have thus verified the claim. But this means that $n$th suspension and $\psi_{-\ell} \circ \check{\Gamma} \circ \theta_{\ell}$ induce homotopic maps on the $K$-theory space of (Perf $\left.(P)^{[\ell-1]}, h_{[\ell]}\right)$ so that $\check{\Gamma}^{\circ} \circ \theta_{\ell}$ induces a map of $K$-theory spaces which is injective on homotopy groups.

This proves that $K\left(\operatorname{Perf}(P)^{[\ell-1]}, h_{[\ell]}\right) \simeq K(R)$ via the functor $\Gamma \circ \theta_{\ell}$. The resulting fibration sequence

$$
\tilde{K}(P,[\ell]) \longrightarrow K(P,[\ell-1]) \stackrel{\check{\Gamma} \circ \theta_{\ell}}{\longrightarrow} K(R)
$$

has a section up to homotopy and up to sign induced by $\psi_{-\ell}$ as the composition $\check{\Gamma} \circ \psi_{\ell} \circ \psi_{-\ell}$ is weakly equivalent to $n$th suspension, just as argued above.

We are now in a position to return to the main theorem of the paper. 
Theorem 1.5.1 Let $P \subseteq \mathbb{R}^{n}$ be an $n$-dimensional lattice polytope, and let $n_{P}$ be the number of distinct integral roots of its EHRHART polynomial $E_{P}(x)$. Let $R$ be a ring with unit; suppose that $R$ is commutative, or else left NOETHERian. Then there is a homotopy equivalence of $K$-theory spaces

$$
K(P) \simeq K(R)^{1+n_{P}} \times K\left(P,\left[n_{P}\right]\right)
$$

where $K(R)$ denotes the $K$-theory of the ring $R$, and $K\left(P,\left[n_{P}\right]\right)$ denotes the $K$-theory of those perfect complexes $Y \in \operatorname{Perf}(P)$ which satisfy $\check{\Gamma}(Y(j)) \simeq 0$ for $0 \leqslant j \leqslant n_{P}$. If $n_{P}=0$ this expresses the tautological splitting $K(P) \simeq K(R) \times \tilde{K}(P)$ where $\tilde{K}(P)=K(P,[0])$.

Proof This follows by concatenating the homotopy equivalences from Propositions 3.1.1 and 3.2.1 for $\ell=1,2, \ldots, n_{p}$.

\subsection{Algebraic $K$-theory of projective space}

Theorem 3.3.1 Let $\Delta^{n}$ be an $n$-dimensional simplex with volume $1 / n !$. Then $n_{\Delta^{n}}=n$ and $K\left(\Delta^{n},[n]\right) \simeq *$ so there is a homotopy equivalence

$$
K\left(\Delta^{n}\right) \simeq K(R)^{n+1}
$$

Let us remark first that an $n$-dimensional simplex with volume $1 / n$ ! can be transformed, by integral translation and a linear map in $G L_{n}(\mathbb{Z})$, into the standard simplex with vertices $0, e_{1}, \ldots, e_{n} \in \mathbb{R}^{n}$. Up to isomorphism, the algebraic data associated to $\Delta^{n}$ does not change so that we may assume $\Delta^{n}$ to be a standard simplex to begin with. Its EHRHART polynomial is $E_{\Delta^{n}}(x)=(x+1)(x+2) \cdots(x+n) / n$ ! which has precisely $n_{\Delta^{n}}=n$ integral roots.

In case of a commutative ring $R$ we have $X_{\Delta^{n}}=\mathbb{P}_{R}^{n}$, projective $n$-space over $R$, and the splitting of Theorem 3.3.1 corresponds to the known splitting of $K$-theory of projective $n$-space which in turn is a special case of QUILLEN's "projective bundle" theorem in $K$-theory applied to the trivial vector bundle of rank $n+1$ over the affine scheme Spec $R$ [8, Sect. 8.2] [11, 4.1].

Proof of 3.3.1 It is enough to prove the following assertion:

3.3.1.1 Let $Y \in \operatorname{hCoh}\left(\Delta^{n}\right)$ be such that $\check{\Gamma}(Y(\ell))$ is acyclic for all $\ell$ with $0 \leqslant \ell \leqslant n$. Then the chain complexes $Y^{F}$ are acyclic for all $F \in F\left(\Delta^{n}\right)_{0}$.

For then the map $Y \longrightarrow 0$ in hCoh $\left(\Delta^{n}\right)$ gives a weak equivalence of endo-functors of Perf $(P)^{[n]}$ from the identity to the zero functor. Consequently, the identity map of $K\left(\Delta^{n},[n]\right)$ is null homotopic so that $K\left(\Delta^{n},[n]\right) \simeq *$. The theorem now follows from the splitting result 1.5.1 and the fact that $n_{\Delta^{n}}=n$.

The above assertion roughly states that the sheaves $\mathcal{O}(k), 0 \leqslant k \leqslant n$, generate the derived category of hCoh $(P)$. This point of view has been pursued, in a model category context, in $[6,3.3 .5]$. We sketch the argument for the reader's convenience. 
Suppose $Y \in \operatorname{hCoh}\left(\Delta^{n}\right)$ has the property that all the structure maps $Y^{F} \longrightarrow Y^{G}$ are quasi-isomorphisms, for all pairs $F \subseteq G$ of non-empty faces of $\Delta^{n}$. For fixed $F \in F\left(\Delta^{n}\right)_{0}$ the structure maps then induce a chain of quasi-isomorphisms of diagrams

$$
Y \longrightarrow \operatorname{con} Y^{\Delta^{n}} \longleftarrow \operatorname{con} Y^{F} \text {. }
$$

By Proposition 2.2.4 and Lemma 2.7.3 we obtain quasi-isomorphisms of chain complexes of $R$-modules

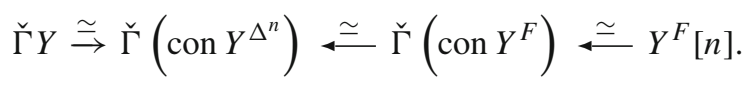

So if in addition $\check{\Gamma}(Y)=\check{\Gamma}(Y(0))$ is acyclic we know that $Y^{F}[n]$ and hence $Y^{F}$ is acyclic as well. - It is thus sufficient to prove the following claim:

3.3.1.2 Let $Y \in \operatorname{hCoh}\left(\Delta^{n}\right)$ be such that $\check{\Gamma}(Y(\ell))$ is acyclic for all $\ell$ with $0 \leqslant \ell \leqslant n$. Then the structure maps $Y^{F} \longrightarrow Y^{G}$ are quasi-isomorphisms for all pairs $F \subseteq G$ of non-empty faces of $\Delta^{n}$.

It is in fact enough to consider structure maps of the form $Y^{F} \longrightarrow Y^{v \vee F}$ for $F \in F\left(\Delta^{n}\right)_{0}$ and a vertex $v$ of $P$.

As remarked above, $\Delta^{n}$ is isomorphic to a standard $n$-simplex with vertices $0, e_{1}, \cdots, e_{n} \in \mathbb{R}^{n}$; the isomorphism can be chosen to map any vertex of $\Delta^{n}$ to 0 . In view of this symmetry it is enough to prove the following:

3.3.1.3 Suppose that $\Delta^{n}$ is a standard $n$-simplex, and suppose that $Y \in \mathrm{hCoh}\left(\Delta^{n}\right)$ is such that $\check{\Gamma}(Y(\ell))$ is acyclic for all $\ell$ with $0 \leqslant \ell \leqslant n$. Then for every face $F$ of $\Delta^{n}$ not containing 0 the structure map $Y^{F} \longrightarrow Y^{0 \vee F}$ is a quasi-isomorphism.

This assertion is proved by induction on $n$, the case $n=0$ being trivial as $\operatorname{Pre}\left(\Delta^{0}\right)=$ $\operatorname{hCoh}\left(\Delta^{0}\right)=\mathrm{Ch}(R$-Mod $)$.

So let $n>0$. For every face $F$ of $\Delta^{n}$ there is an obvious inclusion of sets $k F+T_{F} \subseteq$ $(k+1) F+T_{F}, 0 \leqslant k<n$, which is an equality if and only if $0 \in F$. Hence we have corresponding maps $\mathcal{O}(k) \longrightarrow \mathcal{O}(k+1)$ and $Y(k) \longrightarrow Y(k+1)$ which are identities if $0 \in F$. We obtain short exact sequences in Pre $\left(\Delta^{n}\right)$

$$
0 \longrightarrow Y(k) \longrightarrow Y(k+1) \longrightarrow Z(k+1) \longrightarrow 0
$$

where $Z(k+1)$ is, a priori, simply a name for the cokernel. However, since taking cokernels commutes with tensor products we see that $Z(k+1)$ is indeed the $k$ th twist of $Z(1)=$ coker $(\mathcal{O}(0) \longrightarrow \mathcal{O}(1))$. As the functor $\check{\Gamma}$ preserves short exact sequences we conclude that $\check{\Gamma} Z(k+1)$ is acyclic for $0 \leqslant k<n$ since $\check{\Gamma} Y(k)$ and $\check{\Gamma} Y(k+1)$ are acyclic in this range by hypothesis.

We now have to analyse the diagram $Z(k+1)$ in more detail. If $0 \in F$ the map $Y(k)^{F} \longrightarrow Y(k+1)^{F}$ is the identity, as remarked above, so $Z(k+1)^{F}=0$. Suppose now that $F$ is a face of $\Delta^{n}$ with $0 \notin F$. There is in fact a maximal face $\Delta^{n-1}$ of $\Delta^{n}$ not containing 0 , and $F$ is a face of $\Delta^{n-1}$. We will argue that $Z(k+1)$, when 
restricted to $F\left(\Delta^{n-1}\right)_{0}$, is naturally an object of hCoh $\left(\Delta^{n-1}\right)$ with $\check{\Gamma}_{\Delta^{n-1}} Z(k+1)$ being acyclic for $0 \leqslant k<n$. By induction this implies:

3.3.1.5 The chain complex $Z(1)^{F}$ is acyclic for all $F \in F\left(\Delta^{n-1}\right)_{0}$ and hence for all $F \in F\left(\Delta^{n}\right)_{0}$

First let $\mathbb{R}^{n-1}$ denote the affine hull of $\Delta^{n-1}$, turned into a vector space by distinguishing a lattice point as origin. It comes equipped with its own integer lattice $\mathbb{Z}^{n-1}=\mathbb{Z}^{n} \cap \mathbb{R}^{n-1}$. Let $F \in F\left(\Delta^{n-1}\right)_{0}$. Then

$$
\left(\left((k+1) F+T_{F}\right) \cap \mathbb{Z}^{n}\right) \backslash\left(\left(k F+T_{F}\right) \cap \mathbb{Z}^{n}\right)=\left((k+1) F+T_{F}^{\mathbb{R}^{n-1}}\right) \cap \mathbb{Z}^{n-1}
$$

the barrier cones on the left being computed in $\mathbb{R}^{n}$, the barrier cone on the right being computed in $\mathbb{R}^{n-1}$. This translates into an isomorphism

$$
\operatorname{coker}\left(\mathcal{O}_{\Delta^{n}}(k)^{F} \longrightarrow \mathcal{O}_{\Delta^{n}}(k+1)^{F}\right) \cong \mathcal{O}_{\Delta^{n-1}}(k+1)^{F}
$$

By considering $k=-1$ we obtain from this an algebra isomorphism

$$
A_{F}^{\Delta^{n}} / \mathcal{O}_{\Delta^{n}}(-1)^{F}=\mathcal{O}_{\Delta^{n}}(0)^{F} / \mathcal{O}_{\Delta^{n}}(-1)^{F} \cong A_{F}^{\Delta^{n-1}}
$$

and thus an algebra epimorphism $A_{F}^{\Delta^{n}} \longrightarrow A_{F}^{\Delta^{n-1}}$. The isomorphism displayed in (3.3.1.7) is used to equip $Z(k+1)^{F}$ with a natural $A_{F}^{\Delta^{n-1}}$-module structure while the isomorphism (3.3.1.6) is used to verify that twisting with respect to $\Delta^{n}$ and $\Delta^{n-1}$, respectively, is compatible. A straightforward 5-lemma argument shows that $Z(k)$, when considered as an object of Pre $\left(\Delta^{n-1}\right)$, is indeed a homotopy sheaf. Finally, from the definition of ČECH complexes it follows that the chain complexes $\check{\Gamma}_{\Delta^{n}}(Z(k+1))$ and $\check{\Gamma}_{\Delta^{n-1}}\left(\left.Z(k+1)\right|_{F\left(\Delta^{n-1}\right)_{0}}\right)$ agree up to re-indexing by 1 . In total, this means that $Z(k+1) \in \mathrm{hCoh}\left(\Delta^{n-1}\right)$ satisfies the induction hypotheses as claimed. We have thus verified 3.3 .

From the short exact sequence (3.3.1.4), restricted to $F$-components, we infer that the map $\sigma_{F}: Y(0)^{F} \longrightarrow Y(1)^{F}$ is a quasi-isomorphism of chain complexes, hence so is the map from $Y(0)^{F}$ to the colimit of the infinite sequence

$$
Y(0)^{F} \underset{\sigma_{F}}{\stackrel{\simeq}{\longrightarrow}} Y(1)^{F} \stackrel{\cong}{\rightarrow} Y(0)^{F} \underset{\sigma_{F}}{\stackrel{\simeq}{\longrightarrow}} Y(1)^{F} \stackrel{\cong}{\rightarrow} Y(0)^{F} \stackrel{\simeq}{\rightarrow} \ldots
$$

Here every second map is a fixed isomorphism between $Y(0)^{F}$ and $Y(1)^{F}$. It is not difficult to see that the colimit of this sequence is isomorphic to $A_{0 \vee F} \otimes_{A_{F}} Y^{F}$ (this follows from the fact that the cone $T_{0 \vee F}$ is obtained from the cone $T_{F}$ by forming MINKOWSKI sum with a single ray spanned by the negative of a vector in $T_{F} \cap \mathbb{Z}^{n}$ ). Now the composite

$$
Y^{F} \longrightarrow A_{0 \vee F} \otimes_{A_{F}} Y^{F} \longrightarrow Y^{0 \vee F}
$$


is a structure map of $Y$, and both constituent maps are quasi-isomorphisms: the first by what we have just shown, the second by the stipulation that $Y$ be a homotopy sheaf. In total, we have verified that the structure map $Y^{F} \longrightarrow Y^{0 \vee F}$ is a quasi-isomorphism. We are done.

\section{References}

1. Barvinok, A.: Integer points in polyhedra, Zurich Lectures in Advanced Mathematics. European Mathematical Society (EMS), Zürich (2008)

2. Fulton, W.: Introduction to toric varieties, Annals of Mathematics Studies, vol. 131. The William H. Roever Lectures in Geometry. Princeton University Press, Princeton (1993)

3. Hartshorne, R.: Algebraic geometry. Graduate Texts in Mathematics, vol. 52. Springer, New York (1977)

4. Hüttemann, T.: Finiteness of total cofibres. K-Theory 31, 101-123 (2004)

5. Hüttemann, T.: $K$-theory of non-linear projective toric varieties. Forum Math. 21(1), 67-100 (2009)

6. Hüttemann, T.: On the derived category of a regular toric scheme. Geom. Dedic. 148, 175-203 (2010)

7. Miller, E., Sturmfels, B.: Combinatorial commutative algebra. Graduate Texts in Mathematics, vol. 227. Springer, New York (2005)

8. Quillen, D.: Higher algebraic $K$-theory. I, Algebr. $K$-Theory I. In: Proc. conf. Battelle Inst. 1972, Lecture Notes in Mathematics, vol. 341, pp. 85-147 (1973)

9. Rosenberg, J.: Algebraic K-theory and its applications. Graduate Texts in Mathematics, vol. 147. Springer, New York (1994)

10. Stanley, R.P.: Two poset polytopes. Discret. Comput. Geom. 1(1), 9-23 (1986)

11. Thomason, R.W., Trobaugh, T.: Higher algebraic $K$-theory of schemes and of derived categories. The GROTHENDIECK Festschrift. A collection of articles written in honor of the 60th birthday of Alexander Grothendieck, Birkhäuser, Boston, pp. 247-435 (1990)

12. Waldhausen, F.: Algebraic $K$-theory of spaces. Algebraic and geometric topology. In: Proc. conf., New Brunswick, Lecture Notes in Mathemetics, vol. 1126, pp. 318-419 (1985)

13. Weibel, C.A.: An introduction to homological algebra. Cambridge Studies in Advanced Mathematics. Cambridge University Press, Cambridge (1995) 